\title{
Equilibrium Signaling: Molecular Communication Robust to Geometry Uncertainties
}

\author{
Bayram Cevdet Akdeniz, Malcolm Egan and Bao Quoc Tang
}

\begin{abstract}
A basic property of any diffusion-based molecular communication system is the geometry of the enclosing container. In particular, the geometry influences the system's behavior near the boundary and in all existing modulation schemes governs receiver design. However, it is not always straightforward to characterize the geometry of the system. This is particularly the case when the molecular communication system operates in scenarios where the geometry may be complex or dynamic. In this paper, we propose a new scheme-called equilibrium signaling-which is robust to uncertainties in the geometry of the fluid boundary. In particular, receiver design only depends on the relative volumes of the transmitter or receiver, and the entire container. Our scheme relies on reversible reactions in the transmitter and the receiver, which ensure the existence of an equilibrium state into which information is encoded. In this case, we derive near optimal detection rules and develop a simple and effective estimation method to obtain the container volume. We also show that equilibrium signaling can outperform classical modulation schemes, such as concentration shift keying, under practical sampling constraints imposed by biological oscillators.
\end{abstract}

\section{INTRODUCTION}

The motion of molecules in a fluid depends on many factors including the composition of the fluid, the possibility of chemical reactions, the presence of external forces that may induce drift, molecular degradation, the presence of obstacles, and the structure of the fluid boundary. Pioneering work beginning with Einstein, Langevin and Smoluchowski [1] has established a range of stochastic models to describe how the quantity of molecules varies over time due to diffusion, reaction and convection. Unfortunately, it is challenging to obtain tractable solutions to the equations governing motion, except in a few special cases, such as free diffusion. One of the major difficulties in obtaining tractable solutions is the presence of boundary conditions. That is, the geometry of the fluid boundary including its shape and the extent that it reflects or absorbs molecules.

The lack of tractable solutions to the equations of molecular motion with non-trivial boundary conditions has important implications for molecular communications. For many schemes-most notably concentration shift keying (CSK) [2][14]-receiver design relies heavily on the statistics for the number of molecules in the receiver at a given sampling time. As these statistics are obtained from the equations of motion, a lack of tractable solutions increases the complexity

B.C. Akdeniz was with Université de Lyon, INSA Lyon, INRIA, CITI and he is now with the Centre for Bioinformatics at University of Oslo, M. Egan is with Université de Lyon, INSA Lyon, INRIA, CITI, France. B.Q. Tang is with Insitute of Mathematics and Scientific Computing, University of Graz, Austria of the receiver and, due to the use of potentially inaccurate approximate solutions, can reduce system performance.

At the same time, solutions to the equations of motion require estimates for the parameters of the system. In the case the fluid boundary of the system or container has a complex shape or the diffusion coefficient of the fluid is not well-characterized, the resulting receiver may not be robust, again leading to performance reductions. Moreover, as receiver design relies on solutions for the equations of motion at a particular location, imperfect estimation of the distance between the transmitter and receiver also degrades performance. While there has been active work on distance estimation (see, e.g., [15]), it remains a challenging problem when the transmitter and receiver are themselves moving.

In this paper, we propose a new signaling scheme, called equilibrium signaling, tailored to systems with a finite, reflective fluid boundary as well as reversible reactions-which can be viewed as signaling pathways in biological systems [16]in the transmitter and a passive receiver. A key feature of our scheme is that it can improve the robustness of molecular communication to uncertainties in the system boundary, locations of devices, and also diffusion coefficients. This is the case even if the diffusion coefficients are spatially inhomogeneous-i.e., are not constant throughout the fluid medium-which arises in many biological systems [17]. For example, equilibrium signaling can be readily applied in the scenarios with nonstandard fluid boundaries illustrated in Fig. 1a and Fig. 1b, which is challenging for standard approaches such as CSK, MoSK [18]-[20] and RSK [21].

The source of robustness in equilibrium signaling is that, under the assumptions detailed and justified in the sequel, the receiver observes molecules in a state near equilibrium. Unlike in previously proposed schemes, such as CSK, (MoSK) or RSK, the statistics of the receiver observations only depend on the quantity of molecules emitted, reaction rates in the transmitter and receiver, and the volume of the container. As such, the precise values of the diffusion coefficients, the shape or geometry of the reflective fluid boundary, and the locations of the transmitter and receiver are not required to reliably detect transmitted symbols.

In order to exploit equilibrium signaling, it is necessary to wait for the system to reach a state near equilibrium before sampling after a new transmission. As such, the required symbol period is typically longer than that of CSK, MoSK or RSK. Nevertheless, realistic systems require an oscillator to perform sampling. When a biological oscillator is utilized, its period normally exceeds one minute [22]. In this case, the system may reach an approximate equilibrium state simply 


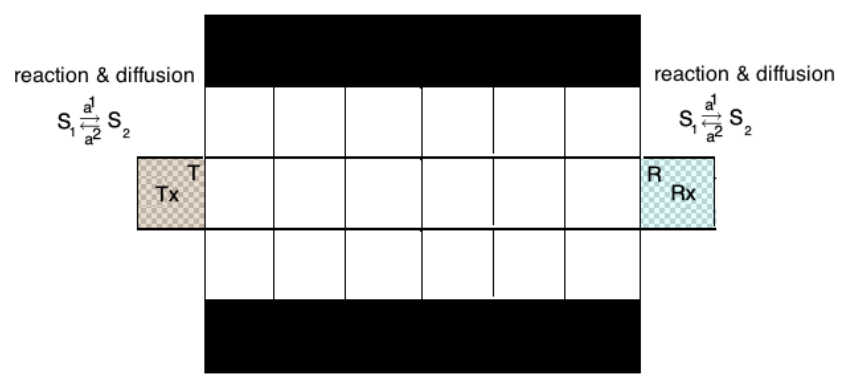

(a) A 2-D channel (molecules cannot diffuse into the black region).

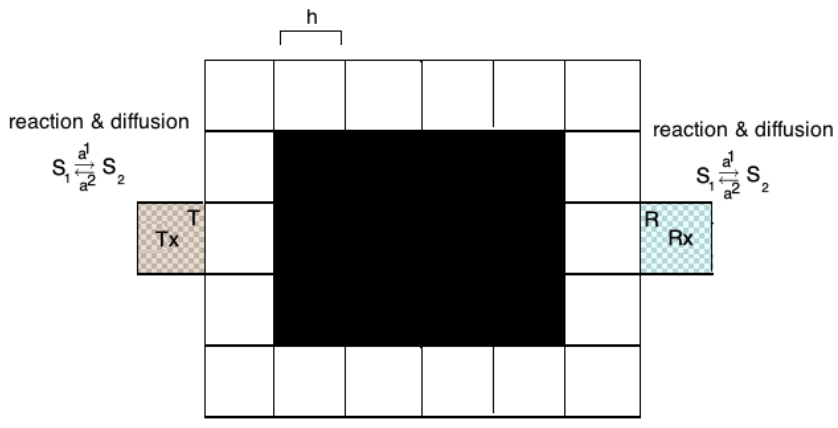

(b) A 2-D channel (molecules cannot diffuse into the black region).

Fig. 1: Fluid boundaries with complex geometry.

due to limitations of the oscillator.

\section{A. Related Work}

Equilibrium signaling bears many similarities to CSK in that both schemes transmit information by varying the quantity of emitted molecules. One key difference is in the choice of the sampling time: in CSK, the sampling time is typically optimized in order to maximize the average number of molecules to arrive in the receiver; while in equilibrium signaling, the sampling time is chosen such that the system approximately reaches equilibrium. A key requirement for CSK is therefore that information carrying molecules are able to leave the fluid medium; either by being absorbed by the transmitter as in the vast majority of CSK schemes [2]-[12], [14], reacting with substrates in the channel as in [23], [24], or diffusing far away when no boundary is present as in [13]. Moreover, CSK also requires the full solution of the Fokker-Planck equation governing molecular motion [25], which is computationally expensive in complex channels, such as in Fig. 1a and Fig. 1b. The computational burden is also increased by the fact that new solutions to the Fokker-Planck equation may be required whenever the transmitter and receiver change their locations.

On the other hand, equilibrium signaling relies on molecules remaining in the system in order for the equilibrium state to be reached. As such, molecules cannot degrade (or at least, must degrade slowly), the receiver is passive, and the fluid boundary is reflective. A typical application of equilibrium signaling is therefore in the context of biological systems enclosed by a membrane, or in microfluidic systems exploiting microfiltration [26]. Indeed, we do not expect equilibrium signaling to play a role in, for example, communication through blood vessels where drift is induced by a linear potential [27].

Equilibrium signaling, as developed in this paper, also bears superficial similarities to MoSK [18] and schemes known as reactive signaling [23], where two species of molecules are employed. In binary MoSK schemes, a different molecule is used to transmit each symbol. On the other hand, reactive signaling transmits two different molecules-which can react-into the channel in order to mitigate inter-symbol interference. While equilibrium signaling also utilizes two different molecules, only one can pass into the channel. That is, only one of the molecules can react to form the other, as occurs in isomerization [28] or via enzyme-aided reactions [24]. As such, the chemical reactions considered in equilibrium signaling may be viewed as a signaling pathway. We also highlight that, like CSK, MoSK and reactive signaling also require a full solution of the Fokker-Planck equation, which is computationally expensive.

Another family of schemes is that of RSK [21]. In RSK, a continuous-time molecular signal is transmitted and detected via chemical reactions. In common with equilibrium signaling is the use of chemical reactions in the transmitter and receiver; however, like CSK and MoSK, RSK does not exploit an equilibrium state and requires computation of the expected quantity of molecules in the receiver at a given time. As this requires a full solution of the equation of motion, good estimates of transmitter-receiver distances and diffusion coefficients are implicitly required. This again contrasts with equilibrium signaling — which also has a dramatically lower complexity-where these estimates are not required.

\section{B. Main Contributions}

We highlight the following main contributions of this paper:

(i) We propose a new signaling scheme for molecular communications, which exploits the presence of an equilibrium state in the system for detection. Under the assumptions detailed in Sec. II, equilibrium signaling is robust to uncertainty in the geometry of a finite, reflective fluid boundary, location of the transmitter and receiver, as well as diffusion coefficients.

(ii) We derive an accurate closed-form Gaussian approximation for receiver observations, which yields a highperformance receiver based on the Viterbi algorithm. We highlight that our scheme accounts explicitly for intersymbol interference.

(iii) We develop a low-memory sub-optimal receiver, again accounting for inter-symbol interference, at the cost of a small loss in performance in terms of the average probability of error. Our low-memory scheme performs symbol-by-symbol detection, analogous to that proposed for standard CSK modulation (see e.g., [29], [30]).

(iv) We provide a simple method to estimate the system volume in the receiver observation statistics, which is the main potential source of uncertainty. We also derive a tractable approximation for the required sampling 
time to ensure that the system is approximately in an equilibrium state.

(v) Via particle-based simulations, we evaluate the average probability of error in our scheme and study the impact of using only a single molecule, analogous to CSK. The simulations show that exploiting equilibrium signaling with chemical reactions in the transmitter and receiver can significantly improve performance over schemes exploiting only a single molecule.

\section{Structure of the Paper}

In Sec. II, we detail the key assumptions in our model and justify its biological relevance. In particular, we set up the reaction-diffusion master equation, which governs the evolution of the statistics for the quantity of molecules in each part of the system. In Sec. III, we develop the equilibrium signaling approach and two detection algorithms tailored to the induced receiver statistics. In Sec. IV, we provide theoretical and empirical evidence for the receiver statistics exploited by the detection algorithms in Sec. III. In Sec. V, we characterize the performance of the equilibrium signaling approach and optimize system parameters to reduce the probability of error. We also develop a simple estimation procedure for the container volume, the main unknown parameter of the receiver statistics. In Sec. VI, we study via particle-based simulations the average probability of error for equilibrium signaling. In Sec. VII, we conclude by discussing the potential to relax the assumptions in Sec. II and other variations on equilibrium signaling.

TABLE I: Notation.

\begin{tabular}{|c|c|}
\hline Variable & Definition \\
\hline$N$ & Number of voxels in the system. \\
\hline$V_{\text {vox }}$ & Volume of each voxel. \\
\hline$V_{\mathrm{Tx}}, V_{\mathrm{Rx}}$ & Volume of the transmitter and receiver. \\
\hline $\mathrm{S}_{1}, \mathrm{~S}_{2}$ & Chemical species. \\
\hline $\mathbf{M}_{i}(t)=\left[M_{i}^{1}(t), M_{i}^{2}(t)\right]$ & State vector of voxel $i$ in time $t$. \\
\hline$\kappa_{i j}^{l}$ & Diffusive jump rate. \\
\hline$a_{i}^{l}, l=1,2$ & Reaction rate constants. \\
\hline$\nu_{l, k}$ & $\begin{array}{l}\text { Quantity of each species } l \\
\text { produced or removed in reaction } k \text {. }\end{array}$ \\
\hline$N_{\mathrm{Rx} j}(t)$ & $\begin{array}{l}\text { Number of } S_{j} \text { molecules in the } \\
\text { receiver at time } t \text {. }\end{array}$ \\
\hline$S_{n}^{m}$ & $\begin{array}{l}\text { Binary sequence with length } n \text { and } \\
m \text { elements bit } 1 .\end{array}$ \\
\hline$s_{k}$ & $k$-th symbol of $S_{n}^{m}$. \\
\hline$T_{s}$ & Communication time interval. \\
\hline$\Delta$ & $\begin{array}{l}\text { Number of transmitted molecules } \\
\text { for each bit } 1 \text { transmission. }\end{array}$ \\
\hline$\mu_{r}$ & $\begin{array}{l}\text { Expected number of molecules in the } \\
\text { receiver after a single bit- } 1 \text { transmission: } \\
\mu_{r}=\mu_{r, 1} \text { if molecules of species } \mathrm{S}_{1} \\
\text { are observed; and } \mu_{r}=\mu_{r, 1}+\mu_{r, 2} \\
\text { if both } \mathrm{S}_{1} \text { and } \mathrm{S}_{2} \text { are observed. }\end{array}$ \\
\hline$D_{l}(\mathbf{x}), l=1,2$. & Spatially dependent diffusion coefficient. \\
\hline
\end{tabular}

\section{System Model}

Let $\Omega \subset \mathbb{R}^{d}, d \in\{1,2,3\}$ be a domain with smooth boundary $\partial \Omega$ consisting of transmitting and receiving devices (corresponding to the subdomain $\Omega_{S} \subset \Omega$ ) with a fluid medium separating the devices. Consider the discretization of $\Omega$ into $N$ volume elements (voxels) each of volume $V_{\text {vox }}$. Here, volume is interpreted as length in $\mathbb{R}^{1}$, area in $\mathbb{R}^{2}$, and volume in $\mathbb{R}^{3}$.

Messages to be sent by the transmitter with volume $V_{\mathrm{Tx}}$ are encoded into the quantity of species $S_{1}$. Within the transmitter and the receiver, each species is produced or removed via the unimolecular reactions

$$
\begin{aligned}
& \mathrm{S}_{1} \rightarrow \mathrm{S}_{2} \\
& \mathrm{~S}_{2} \rightarrow \mathrm{S}_{1} .
\end{aligned}
$$

In particular, the transmitter produces information-carrying molecules of species $S_{2}$ by the first reaction in (1). We note that the reactions in (1) can be interpreted as a signaling pathway, which are ubiquitious in biological systems [16]. For example, the reactions in (1) may approximate the more general family

$$
\begin{aligned}
& \mathrm{S}_{1} \rightarrow \mathrm{A}_{1} \rightarrow \cdots \rightarrow \mathrm{A}_{m} \rightarrow \mathrm{S}_{2} \\
& \mathrm{~S}_{2} \rightarrow \mathrm{B}_{1} \rightarrow \cdots \rightarrow \mathrm{B}_{n} \rightarrow \mathrm{S}_{1},
\end{aligned}
$$

under the assumption that the intermediate reactions occur sufficiently rapidly. The reactions in (1) may also be viewed as an approximation of the enzyme-aided system

$$
\begin{aligned}
& \mathrm{S}_{1}+\mathrm{E}_{1} \rightarrow \overline{\mathrm{S}_{1} \mathrm{E}_{1}} \rightarrow \mathrm{S}_{2}+\mathrm{E}_{1} \\
& \mathrm{~S}_{2}+\mathrm{E}_{2} \rightarrow \overline{\mathrm{S}_{1} \mathrm{E}_{2}} \rightarrow \mathrm{S}_{1}+\mathrm{E}_{2},
\end{aligned}
$$

where $\mathrm{E}_{1}, \mathrm{E}_{2}$ are enzymes and $\overline{\mathrm{S}_{1} \mathrm{E}_{1}}, \overline{\mathrm{S}_{2} \mathrm{E}_{2}}$ are intermediate complexes. Such reactions are ubiquitous in the translation step of DNA replication, where the enzyme is viewed as a ribosome molecule [31].

We assume that molecules of species $S_{1}$ produced in the transmitter are not capable of diffusing into the channel, while this is possible for species $S_{2}$. The absence of the species $S_{1}$ in the channel can arise when the transmitter is encapsulated by a membrane, which can prevent diffusion of $S_{1}$ via electrostatic forces or the size of gaps in the membrane. For example, the transmitter may be a cell or, in microfluidic applications, be encapsulated by a microfilter [26].

At the receiver, with volume $V_{\mathrm{Rx}}$ (not necessarily the same as $V_{\mathrm{Tx}}$ ), molecules of species $\mathrm{S}_{2}$ are able to generate molecules of species $S_{1}$ via the second reaction in (1), which can be viewed as another signaling pathway. The receiver can then attempt to decode the transmitted message based on observations of the quantities of species $S_{1}$ and species $S_{2}$ that are present at the sampling time. As for the transmitter, the molecules of $S_{1}$ cannot diffuse out of the receiver due to the presence of a membrane.

In order to capture the effect of small quantities of each chemical species in the system, we consider a stochastic model for the kinetics. To formally describe the scenario, we introduce the following notation. Let $M_{i}^{l}(t), l=1,2, i=$ $1, \ldots, N$ denote the random variable for the number of molecules of species $\mathrm{S}_{1}$ or $\mathrm{S}_{2}$ in voxel $i$ at time $t$. Denote $\mathbf{M}_{i}(t)=\left[M_{i}^{1}(t), M_{i}^{2}(t)\right]$ as the state vector in voxel $i$ and the matrix consisting of all state vectors as $\mathbf{M}(t)=$ $\left[\mathbf{M}_{1}(t), \ldots, \mathbf{M}_{N}(t)\right]$. The probability that $\mathbf{M}(t)$ has value $\mathbf{m}$ at time $t$ conditioned on there being a quantity of molecules $\mathbf{m}_{0}$ in each voxel at time $t=0$ is then denoted by

$$
P(\mathbf{m}, t)=\operatorname{Pr}\left(\mathbf{M}(t)=\mathbf{m} \mid \mathbf{M}(0)=\mathbf{m}_{0}\right),
$$


where $\mathbf{M}(0)$ is the initial quantity of molecules of each species in each voxel.

Since each reaction is unimolecular, it follows that in each reaction the number of molecules of the two species involved can only increase or decrease by one. Let $\mathbf{1}_{i}^{l}$ be the state where the number of molecules in all voxels is zero, except for species $l$ in voxel $i$. That is, $\mathbf{M}(t)+\mathbf{1}_{i}^{l}$ means that the number of molecules of species $l$ in voxel $i$ is increased by one.

A popular model for stochastic kinetics of molecules is the reaction-diffusion master equation (RDME) [32], also utilized in the context of molecular communications in [33]. In this model, the diffusive jump rate is denoted by $\kappa_{i j}^{l}$ for each individual molecules of the $l$-th species moving from voxel $j$ into voxel $i$, with $\kappa_{i i}^{l}=0, i=1, \ldots, N$. In particular, the probability per unit time that a molecule of $S_{l}$ diffuses from voxel $j$ to voxel $i$ at time $t$ is given by $\kappa_{i j}^{l} M_{j}^{l}(t)$.

In general, $\kappa_{i j}^{l}$ depends on $i, j$ and $l$; that is, the probability of a molecule diffusing between two voxels is not spatially homogeneous (diffusive jump rates vary from voxel to voxel). While spatially homogeneous diffusion is a standard assumption in the molecular communications literature, variations in the fluid environment can induce inhomogeneity which we are able to capture within our model; for example, in developing organisms [17]. In particular, the diffusive jump rate out of the boundary voxels is zero, which can be interpreted as a reflective boundary condition. We remark that spatial inhomogeneity of diffusion does not enforce any boundary constraints (other than on the reflective boundary). Nevertheless, if the diffusive jump rates near the boundary of the receiver are small, the flux of probability for molecules of $S_{1}$ into the receiver will be low. This behavior can model the impact of a membrane surrounding the receiver, which is more difficult for molecules to pass through than in the microchannel.

We remark that an alternative stochastic model has recently been studied in the context of reactive signaling which aims to reduce interference by utilizing the reaction of two molecules [23]. A key feature of the RDME model is that it provides information about the statistical dependence in the receiver observations over time, which is not the case for the model in [23]. We also note that spatial homogeneity for the diffusion process is assumed in [23] and in the vast majority of other work on molecular communications. In the case of mass-action kinetics and first-order reactions, the probability per unit time that a molecule of $S_{l}$ in voxel $i$ reacts at time $t$ is given by $a_{i}^{l} M_{i}^{l}(t)$ with rate constants $a_{i}^{l}$. In general, the reaction rate is dependent on the voxel index. To model production of $S_{1}$ in the transmitter and $S_{2}$, we assume that for voxels $i$ comprising the transmitter and the receiver $a_{i}^{l}=a^{l}$, for $l \in\{1,2\}$, while $a_{i}^{1}=0$ for voxels comprising the channel. Due to the small scale of the transmitter and receiver, we expect homogeneity in key parameters such as temperature, which implies few variations in the reaction rate within the transmitter and the receiver. The net change of each chemical species due to the reaction with substrate $S_{l}$ is expressed via the vector $\boldsymbol{\nu}_{l}=\left[\nu_{l, 1}, \nu_{l, 2}\right] \in \mathbb{N}^{2}$, where $\nu_{l, 1}$ is the net gain of molecules of $S_{1}$ and $\nu_{l, 2}$ is the net gain of molecules of $S_{2}$ in the reaction where $S_{l}$ is the substrate. The term $\boldsymbol{\nu}_{l} \mathbf{1}_{i}$ indicates that $\mathbf{M}(t)$ changes by $\boldsymbol{\nu}_{k}$ in the $i$-th voxel.

In the RMDE model, the probability distribution $\operatorname{Pr}(\mathbf{m}, t)$ evolves according to the system of differential equations given by

$$
\begin{aligned}
& \frac{\mathrm{d} P(\mathbf{m}, t)}{\mathrm{d} t}= \\
& \sum_{i=1}^{N} \sum_{j=1}^{N} \sum_{l=1}^{2}\left(\kappa_{i j}^{l}\left(m_{j}^{l}+1\right) P\left(\mathbf{m}+\mathbf{1}_{j}^{l}-\mathbf{1}_{i}^{l}, t\right)-\kappa_{j i}^{l} m_{i}^{l} P(\mathbf{m}, t)\right) \\
& \quad+\sum_{i=1}^{N} \sum_{l=1}^{2}\left(a_{i}^{l}\left(m_{i}^{l}+1\right) P\left(\mathbf{m}-\boldsymbol{\nu}_{l} \mathbf{1}_{i}, t\right)-a_{i}^{l} m_{i}^{l} P(\mathbf{m}, t)\right),
\end{aligned}
$$

where the first triple sum corresponds to the net increase in probability per unit time due to diffusion and the second double sum corresponds to the net increase in probability per unit time due to chemical reactions. We highlight that implicitly in (5), it is assumed that there is a finite, reflective boundary; no degradation of molecules; constant temperature; and no external forces (e.g., due to a linear potential inducing drift). For more details, we refer the reader to [25].

The system of ordinary differential equations in (5) corresponds to the Kolmogorov forward equation for a continuoustime Markov chain; that is, the evolution of the system state is Markovian. In our setting, due to the reversible reactions and diffusive jump rates detailed above, the Markov chain corresponding to the RDME is irreducible and positive recurrent. Therefore, a stationary distribution exists and is given by [34]

$$
\pi(\mathbf{m})=\lim _{t \rightarrow \infty} \operatorname{Pr}\left(\mathbf{M}(t)=\mathbf{m} \mid \mathbf{M}(0)=\mathbf{m}_{0}\right) .
$$

In summary, we make the following assumptions in this paper:

(i) the fluid boundary or container is smooth, reflective, and with finite volume;

(ii) no external forces are present (e.g., the forces with linear potential that induce drift);

(iii) no chemical reactions are present, with the exception of those detailed in Sec. II;

(iv) molecules do not degrade;

(v) diffusion may be spatially inhomogeneous;

(vi) and motion is governed by the reaction-diffusion master equation.

Certain assumptions can be relaxed while still allowing for equilibrium signaling to be utilized. Such relaxations will be discussed further in Sec. VII.

\section{Proposed Equilibrium Signaling Strategy}

The existence of an equilibrium state provides the opportunity to develop a new signaling strategy. In particular, if the statistics for the quantity of each species at the receiver can be characterized, transmitted symbols may be recovered based on observations near the equilibrium state. As we will show, such an approach is highly robust to uncertainties in the container geometry, which is not the case for classical CSK signaling schemes.

In this section, we detail our proposed equilibrium signaling strategy tailored to the model in Section II. We focus on the 
case of binary signaling; that is, for the transmitter to send a bit 1 , it generates $\Delta$ molecules of species $\mathrm{S}_{1}$ within a single voxel of the transmitter. For the case of bit 0 , the transmitter generates zero molecules of species $S_{1}$. Each bit is equally likely to be sent.

Assume that the system operates using time slots with duration $T_{s}$ and that no molecules of species $S_{1}$ nor $S_{2}$ are present in the system at $t=0$. The bit to be transmitted in time slot $n$ is denoted by $s_{n}$. Moreover, molecules that are produced by the transmitter may change the number of each species via the reactions in (1); however, no molecules degrade. As a consequence, inter-symbol interference is present and is accounted for in the detection algorithms developed in Sec. III-A and Sec. III-B.

Consider the $n$-th time slot. Due to the previous $n-1$ transmissions, there are $N_{\mathrm{Tx}, l}\left(n T_{s}\right), l=1,2$ molecules of species $S_{l}$ in the transmitter. At a time $n T_{s}+\delta$ shortly after the beginning of the time slot, the transmitter produces a quantity of $S_{1}$ depending on the bit to be transmitted. In particular,

$$
N_{\mathrm{Tx}, 1}\left(n T_{s}+\delta\right)=\left\{\begin{array}{cc}
N_{\mathrm{Tx}, 1}\left(n T_{s}\right)+\Delta & s_{n}=1 \\
N_{\mathrm{Tx}, 1}\left(n T_{s}\right) & s_{n}=0,
\end{array}\right.
$$

for $\delta>0$ a sufficiently small period of time; that is, $\delta$ is chosen such that no reactions occur nor any molecules diffuse to a voxel outside of the transmitter.

The key idea behind the proposed signaling strategy is that for sufficiently large $T_{s}$, the total number of molecules of species $S_{1}$ and $S_{2}$ in the receiver at the time of sampling will be approximately drawn from the stationary distribution of the RDME. As such, if the stationary distribution is known, then near-optimal detection rules can be obtained.

To this end, suppose that a sequence of bits, $s_{1}, \ldots, s_{n}$, over a period of $n$ sampling intervals is sent. Let $S_{n}^{m}$ denote such a sequence containing with $m$ transmissions (each corresponding to a bit 1). Further, let $N_{\mathrm{Rx}, 1}\left(n T_{s} \mid S_{n}^{m}\right)$ and $N_{\mathrm{Rx}, 2}\left(n T_{s} \mid S_{n}^{m}\right)$ denote the number of molecules of species $\mathrm{S}_{1}$ and $\mathrm{S}_{2}$, respectively, observed by the receiver at the end of the $n$-th symbol period (i.e., at time $\left.(n+1) T_{s}\right)$, given the transmitted sequence $S_{n}^{m}$.

We make the following assertion, which will be validated in Section IV.

Assertion 1. For $\Delta$ sufficiently large,

$$
\begin{aligned}
& N_{\mathrm{Rx}, 1}\left(n T_{s} \mid S_{n}^{m}\right) \sim \mathcal{N}\left(m \mu_{r, 1}, m \mu_{r, 1}\right), \\
& N_{\mathrm{Rx}, 2}\left(n T_{s} \mid S_{n}^{m}\right) \sim \mathcal{N}\left(m \mu_{r, 2}, m \mu_{r, 2}\right),
\end{aligned}
$$

where $\mu_{r, 1}, \mu_{r, 2}>0$ are known constants, dependent on the volume of the enclosing container and not the specific geometry, and $\mathcal{N}\left(\mu, \sigma^{2}\right)$ denotes the Gaussian law with mean $\mu$ and variance $\sigma^{2}$. In particular,

$$
\mu_{r, 1}=\frac{a^{1}}{a^{2}} \mu_{r, 2}, \quad \mu_{r, 2}=\frac{\Delta \frac{V_{\mathrm{Rx}}}{V_{\mathrm{tot}}}}{1+\frac{a^{1}}{a^{2}} \frac{V_{\mathrm{Tx}}+V_{\mathrm{Rx}}}{V_{\mathrm{tot}}}},
$$

where $V_{\mathrm{tot}}=N V_{\mathrm{Vox}}$ is the total volume of the system. Moreover

$$
\begin{gathered}
N_{\mathrm{Rx}, 1}\left(n T_{s} \mid S_{n}^{m}\right)+N_{\mathrm{Rx}, 2}\left(n T_{s} \mid S_{n}^{m}\right) \sim \\
\mathcal{N}\left(m\left(\mu_{r, 1}+\mu_{r, 2}\right), m\left(\mu_{r, 1}+\mu_{r, 2}\right)\right) .
\end{gathered}
$$

We emphasize that Assertion 1 is not a rigorous statement and for this reason we have called it an assertion rather than a proposition or theorem. For special families of reactiondiffusion systems-e.g., with no diffusion or homogeneous reaction rates and diffusion coefficients) - it is feasible to make Assertion 1 rigorous (see, e.g. [35]). However, to the best of our knowledge it is not possible to directly apply these results to our model.

In (8), $\mu_{r, 1}$ and $\mu_{r, 2}$ are the average number of molecules for corresponding species in each voxel given $\Delta$ molecules are in the system (corresponding to a single transmission of bit 1). To gain some intuition into the values of $\mu_{r, 1}$ and $\mu_{r, 2}$, consider the case when $a^{1}=a^{2}$ and $V_{\mathrm{Rx}}=V_{\mathrm{Tx}}$. Here,

$$
\mu_{r, 1}=\mu_{r, 2}=\frac{\Delta V_{\mathrm{Rx}}}{V_{\mathrm{tot}}+2 V_{\mathrm{Rx}}}
$$

The effect of diffusion is to evenly spread the molecules of each species between all voxels. This implies that the average number of molecules of $S_{1}$ is the same within each voxel comprising $V_{\mathrm{Tx}}$ and $V_{\mathrm{Rx}}$. Moreover, the average number of molecules of $S_{2}$ is the same within each voxel in the total volume. On the other hand, the effect of the reactions is to produce, on average at equilibrium, the same number of molecules of each species within the transmitter and receiver voxels. As such, on average at equilibrium, there are twice the total number of molecules in the transmitter and receiver voxels compared with the voxels comprising the channel from which (11) follows. A similar argument accounting for different reaction rates yields (9).

Suppose that only the quantity of $S_{1}$ is observed by the receiver. Under Assertion 1, the distribution for the quantity of $S_{1}$ in the receiver at the sampling time for the $n+1$-th time slot, corresponding to a transmission $s_{n+1} \in\{0,1\}$ is given by

$$
\begin{aligned}
& N_{\mathrm{Rx}, 1}\left((n+1) T_{s} \mid S_{n}^{m}, s_{n+1}\right) \sim \\
& \left\{\begin{array}{cl}
\mathcal{N}\left(m \mu_{r, 1}, m \mu_{r, 1}\right) & s_{n+1}=0 \\
\mathcal{N}\left((m+1) \mu_{r, 1},(m+1) \mu_{r, 1}\right) & s_{n+1}=1 .
\end{array}\right.
\end{aligned}
$$

Observe in (12) that the impact of previous transmissions is accounted through the parameter $m$, which corresponds to the total number of previous bit- 1 transmissions.

On the other hand, if both $S_{1}$ and $S_{2}$ are observed by the receiver, then the distribution for the total quantity of molecules at the sampling time for the $n+1$-th time slot, corresponding to a transmission $s_{n+1} \in\{0,1\}$ is given by

$$
\begin{aligned}
& N_{\mathrm{Rx}, 1}\left((n+1) T_{s} \mid S_{n}^{m}, s_{n+1}\right) \sim \\
& \left\{\begin{array}{cc}
\mathcal{N}\left(m\left(\mu_{r, 1}+\mu_{r, 2}\right), m\left(\mu_{r, 1}+\mu_{r, 2}\right)\right) & s_{n+1}=0, \\
\mathcal{N}\left((m+1)\left(\mu_{r, 1}+\mu_{r, 2}\right),(m+1)\left(\mu_{r, 1}+\mu_{r, 2}\right)\right) & s_{n+1}=1 .
\end{array}\right.
\end{aligned}
$$

In the following, we will consider the cases where the receiver observes only $S_{1}$, and where both $S_{1}$ and $S_{2}$ are observed. In the first case (only $S_{1}$ ), we define $\mu_{r}=\mu_{r, 1}$. In the second case (both $\mathrm{S}_{1}$ and $\mathrm{S}_{2}$ ), $\mu_{r}=\mu_{r, 1}+\mu_{r, 2}$. 


\section{A. Near-Optimal Detection}

We seek to obtain an estimate for the sequence $\left(s_{1}, \ldots, s_{n+1}\right)$. Although the observation process is Markovian, for a sufficiently large time slot $T_{s}$, the observations are approximately independent. Let $\mathbf{N}_{\mathrm{Rx}}$ denote the vector of observations at the receiver for the quantity of observed molecules (i.e., only $S_{1}$, or $S_{1}$ and $S_{2}$ ) and $\mathbf{s} \in\{0,1\}^{n+1}$ denote a potential vector of transmitted bits. Under Assertion 1, the joint likelihood of the observations is given by

$$
f_{\mathbf{N}_{\mathrm{Rx}} \mid \mathbf{s}}(\mathbf{n})=\prod_{i=1}^{n+1} \frac{\exp \left(-\frac{\left(n_{i}-\mu_{r} \sum_{j=1}^{i} s_{j}\right)^{2}}{2 \mu_{r} \sum_{j=1}^{i} s_{j}}\right)}{\sqrt{2 \pi \mu_{r} \sum_{j=1}^{i} s_{j}}}
$$

and the resulting detection rule is given by

$$
\hat{\mathbf{s}}^{*}=\arg \max _{\mathbf{s} \in\{0,1\}^{n+1}} f_{\mathbf{N}_{\mathrm{Rx}, 1} \mid \mathbf{s}}(\mathbf{n}) .
$$

A brute force search for the estimate $\hat{\mathbf{s}}^{*}$ in (14) leads to a complexity that grows exponentially in $n$. Nevertheless, the Viterbi algorithm with appropriate branch weights can be used to solve the optimization problem with complexity of order $O(n)$. Note that while the Viterbi algorithm yields an optimal solution for (15), it is under the assumption that Assertion 1 holds.

We briefly sketch the computations in Algorithm 1, which is a form of the Viterbi algorithm with branch metrics tailored to the problem in (14). For the $k$-th symbol $s_{k} \in\{0,1\}$, let $p\left(n_{k} \mid s_{k}\right)=\log \left(f_{N_{\mathrm{Rx}}\left(k T_{s}\right) \mid s_{k}}\left(n_{k}\right)\right)$. In the $k$-th symbol interval, it is necessary to compute $P_{k-1,0}$ and $P_{k-1,1}$, which correspond to the probability of the most probable sequence until the $k-1$-th symbol and the $k$-th symbol is 0 and 1 , respectively.

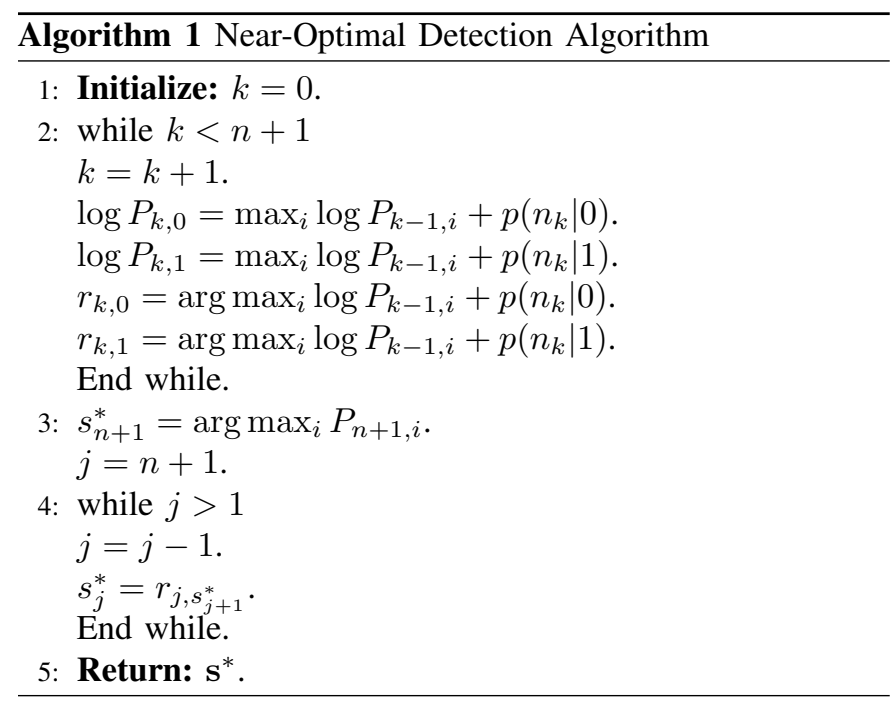

\section{B. Detection with Low Memory Requirements}

For large $n$, directly solving the optimization problem in (15) requires the storage all previous observations, which may not be feasible due to limitations of the underlying biological circuits. As such, it also is desirable to consider approaches that only require limited memory.
To this end, define

$$
R(n+1)=N_{\mathrm{Rx}}\left((n+1) T_{s}\right)-N_{\mathrm{Rx}}\left(n T_{s}\right) .
$$

In this case, each bit is decoded sequentially via the detection rule

$$
\tilde{s}_{n+1}= \begin{cases}1 & R(n+1)>\tau, \\ 0 & \text { otherwise }\end{cases}
$$

The optimal choice of $\tau$ for the decision rule in (17) can be obtained via an analysis of the bit error rate, which we carry out in Section V-B. As will be shown in Section VI via particle-based simulations, this low memory detection achieves nearly the same performance as the near-optimal algorithm in Algorithm 1.

\section{EQUILIBRIUM CHARACTERIZATION: JUSTIFICATION OF ASSERTION 1}

The potential of the signaling scheme in Section III relies on the validity of Assertion 1. We first develop a stochastic linear noise approximation of the RDME model in Section II, which justifies the Gaussian law. We then perform the KolmogorovSmirnov test to provide a further empirical validation.

\section{A. Stochastic Linear Noise Approximation}

It is known that for stochastic chemical reaction networks under mass-action kinetics, the evolution of the molecular counts of each species can be approximated by the chemical Langevin equation [36]. Since diffusion is modeled by unimolecular reactions in the RDME model in Section II, it follows that a similar approximation can be applied. In particular, following [36], we have

$$
\begin{aligned}
& M_{i}^{l}(t+\tau) \approx M_{i}^{l}(t) \\
& +\sum_{j=1}^{N} \kappa_{i j}^{l} M_{j}^{l}(t) \tau+\sum_{j=1}^{N} \sqrt{\kappa_{i j}^{l} M_{j}^{l}(t) \tau} \mathcal{N}_{D, j}(0,1)-\sum_{j=1}^{N} \kappa_{j i} M_{i}^{l}(t) \\
& +\sum_{j=1}^{N} \sqrt{\kappa_{j i}^{l} M_{i}^{l}(t) \tau} \mathcal{N}_{D^{\prime}, j}(0,1)+a_{i}^{3-l} M_{i}^{3-l}(t) \tau-a_{i}^{l} M_{i}^{l}(t) \tau \\
& +\sum_{k=1}^{2} \sqrt{a_{i}^{k} M_{i}^{k}(t) \tau} \mathcal{N}_{R, k}(0,1),
\end{aligned}
$$

where each standard normal random variable $\mathcal{N}_{D, j}(0,1)$, $\mathcal{N}_{D^{\prime}, j}(0,1)$, and $\mathcal{N}_{R, k}(0,1)$ are independent.

Let $V=V_{i}, i=1, \ldots, N$ be the volume of voxel $i$ and define the concentration of species $S_{l}$ in voxel $i$ by

$$
C_{i}^{l}(t)=\frac{M_{i}^{l}(t)}{V} .
$$

It then follows from the RDME that

$$
\begin{gathered}
\left.\frac{\mathrm{d} \mathbb{E}\left[C_{i}^{l}(t)\right]}{\mathrm{d} t}=\sum_{j=1}^{N}\left(\kappa_{i j}^{l} \mathbb{E}\left[C_{j}^{l}(t)\right]-\kappa_{j i}^{l} \mathbb{E}\left[C_{i}^{l}(t)\right]\right)-\mathbb{E}\left[a_{i}^{l} C_{i}^{l}(t)\right)\right] \\
+\mathbb{E}\left[a_{i}^{3-l} C_{i}^{3-l}(t)\right],
\end{gathered}
$$

with details given in [32, Sec. 1.1.3].

Since all reactions are unimolecular, under the assumption that the diffusion jump rates, $\kappa_{i j}$, are chosen appropriately, the 
expected concentrations converge to a deterministic reactiondiffusion system [32]. In particular, let $u_{1}, u_{2}$ be the deterministic concentrations. Then, the deterministic system is described by the system of partial differential equations, for all $l=1,2$,

$$
\begin{cases}\partial_{t} u_{l}-\operatorname{div}\left(D_{l}(\mathbf{x}) \nabla u_{l}\right)=a^{3-l}(\mathbf{x}) u_{3-l}-a^{l}(\mathbf{x}) u_{l}, & \mathbf{x} \in \Omega, \\ D_{l}(\mathbf{x}) \nabla u_{l} \cdot \nu=0, & \mathbf{x} \in \partial \Omega, \\ u_{l}(\mathbf{x}, 0)=u_{l 0}(\mathbf{x}), & \mathbf{x} \in \Omega,\end{cases}
$$

where $\partial_{t}$ denotes the derivative with respect to time. The vector-valued function $\nu(\mathbf{x})$ is the outer unit normal defined for $\mathbf{x} \in \partial \Omega$. The condition $D_{l}(\mathbf{x}) \nabla u_{l} \cdot \nu=0$ is a homogeneous Neumann boundary condition. The initial data $u_{l}, l=1,2$, is assumed to be nonnegative. The diffusion coefficients satisfy $D_{l}(\mathbf{x}) \geq 0$ and can be zero on a set with positive measure. For spatially homogeneous diffusion coefficients $D_{l}(\mathbf{x})=$ $D_{l}, \mathbf{x} \in \Omega$.

Using the same argument as [37], it follows from the Langevin approximation in (18) that fluctuations are of the order of $\sqrt{V}$. This suggests the Gaussian approximation

$$
M_{i}^{l}(t) \approx V u_{l}\left(\mathbf{x}_{i}, t\right)+\sqrt{V} Z_{i}^{l}(t),
$$

where $Z_{i}^{l}(t)$ is a zero-mean Gaussian random variable and $\mathbf{x}_{i}$ is a point inside the $i$-th voxel. We note that this approximation can be rigorously justified in the case of chemical reaction networks [37] via a convergence result in [35]. A similar result for the reaction-diffusion setting with spatially homogeneous diffusion and reactions is available in [38].

\section{B. Verification of Assertion 1}

The linear noise approximation provides a justification for the Gaussianity of the stationary distribution required to establish Assertion 1. However, the mean and the variance are dependent on the equilibrium solution to the system of PDEs in (21). At present, for the spatially inhomogeneous reaction rates and diffusion coefficients, even the existence of an equilibrium solution has not been rigorously established.

Nevertheless, the ansatz for the mean and variance of the stationary distribution in Assertion 1 is provided for the related problem where only the diffusion coefficients are spatially inhomogeneous; that is, the reaction rates are spatially homogeneous or, said in different words, independent of the spatial coordinates. In this case, it has been established in [39] that the mean and variance do indeed correspond to those given in Assertion 1.

To empirically validate the ansatz, we have carried out Monte Carlo simulations and performed a KolmogorovSmirnov test. Both a standard 2-D scenario and a nonstandard 2-D scenario are considered, illustrated in Fig. 2a and Fig. 2b, respectively. In the numerical validation, the following parameters are used: $a^{1}=a^{2}=1, \Delta=600$, $V_{\mathrm{Rx}}=V_{\mathrm{Tx}}=V_{\mathrm{vox}}=10^{-6}, N \in\{60,100\}, D_{2}(\mathbf{x})=D_{2} \in$ $\{4,40,400\} \times 10^{-9}, \mathbf{x} \in \Omega$, the corresponding diffusive jump rates $\kappa_{i j}^{l}=D_{l} / h^{2}$ for cubic voxels with height $h$ [32].

The first step is to verify that the deterministic system of differential equation in (21) admits a spatially homogeneous solution as the time $t \rightarrow \infty$. To do so, we obtain a numerical solution using the method in [40], for a range of different system parameters.

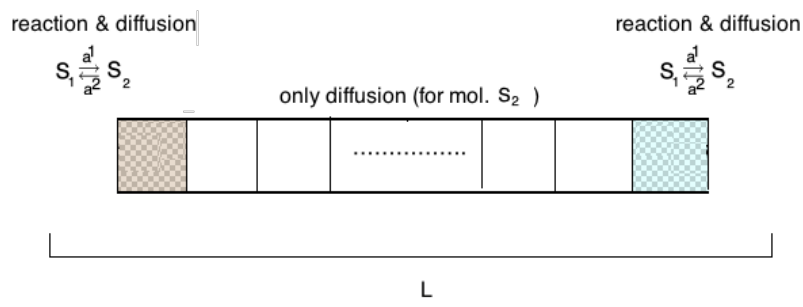

(a) Standard 2-D scenario.

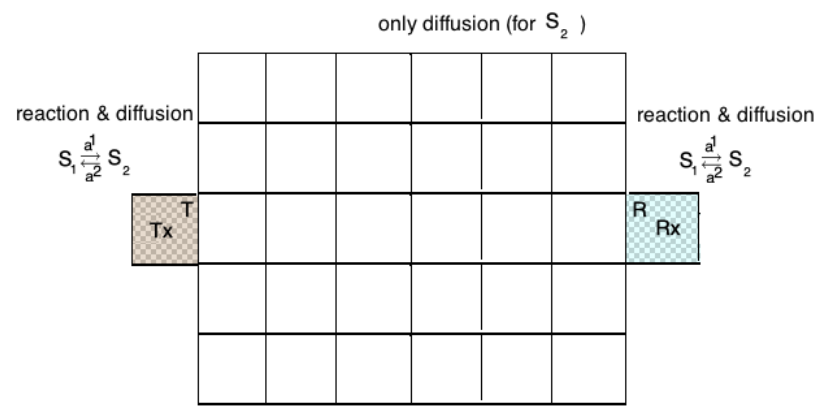

(b) Non-standard 2-D scenario.

Fig. 2: Miscellaneous channel models

To verify that the mean quantity of molecules is consistent with Assertion 1, we estimate the mean from a particle-based simulation. In Fig. 3, there are $N=60$ voxels and $\Delta=600$ molecules in the system. In the voxels of the transmitter and the receiver, both $S_{1}$ and $S_{2}$ are present while $S_{1}$ can only diffuse in the voxels corresponding to the transmitter and the receiver. Moreover, since the reactions $a^{1}=a^{2}$, the expected number of molecules at the equilibrium for each voxel is equal to $\frac{600}{60+2}=9.68$, which is consistent with Fig. 3. In Fig. 4, we examine the scenario where the diffusion coefficient is spatially inhomogeneous and again observe spatial homogeneity of the equilibrium. Here, $N=100$ and hence the expected number of molecules at the equilibrium for each voxel is equal to $\frac{600}{100+2}=5.88$.

In order to verify that the number of observed molecules is well approximated by the Gaussian law in Assertion 1, we perform the Kolmogorov-Smirnov test based on particlebased simulations of the system. In the Kolmogorov-Smirnov test, $H(t)$ denotes be the emprical distribution function (estimated from the simulated data) and $F(t)$ denotes the candidate distribution function (given in Assertion 1). The Kolmogorov-Smirnov statistic between two distributions is then given by $T^{*}=\sup _{t}(|H(t)-F(t)|)$. The hypothesis that the candidate distribution is true is rejected if

$$
\alpha>1-F_{\mathrm{Kol}}\left(\sqrt{U} T^{*}\right),
$$

where $\alpha \in(0,1)$ is the significance level ( $\alpha \approx 0$ corresponds to high significance), $U$ is the number of samples, and $F_{\mathrm{Kol}}$ is the distribution function of Kolmogorov distribution [41]. 


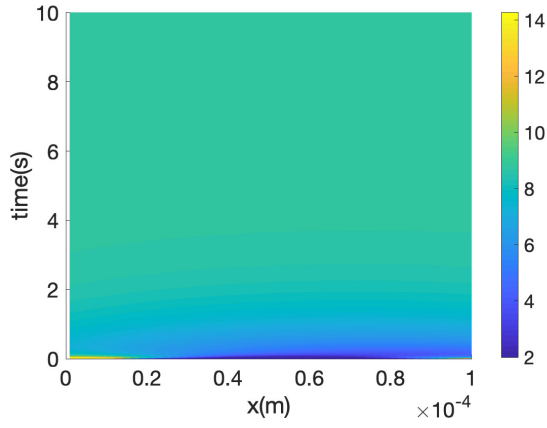

(a) $D_{2}=4 \times 10^{-9} \mathrm{~m}^{2} / \mathrm{s}$ for all $x \in$.

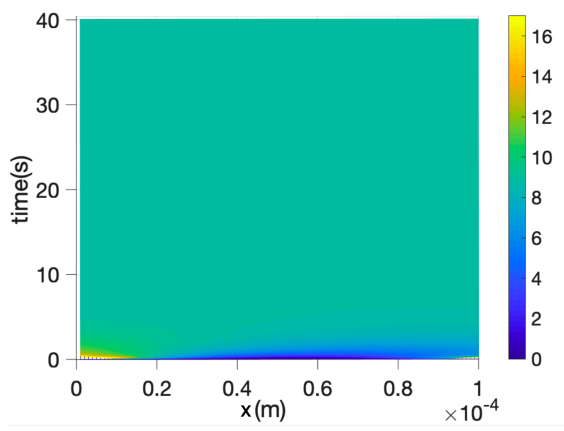

(b) $D_{2}=40 \times 10^{-9} \mathrm{~m}^{2} / \mathrm{s}$.

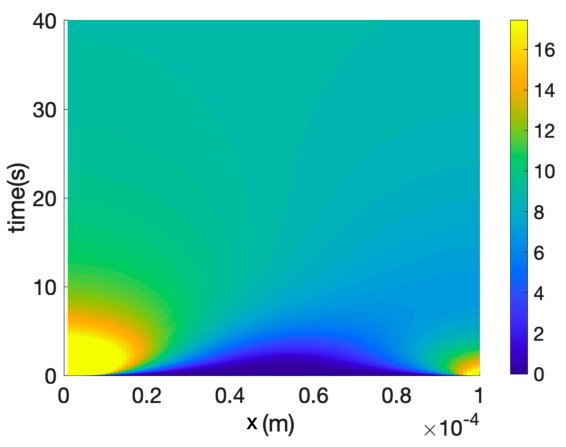

(c) $D_{2}=400 \times 10^{-9} \mathrm{~m}^{2} / \mathrm{s}$.

Fig. 3: Solution of (21) for the concentration of $\mathrm{S}_{2}$ for $a^{1}=a^{2}=1 \mathrm{~s}^{-1}, \Delta=600, V_{\mathrm{Rx}}=V_{\mathrm{Tx}}=V_{\mathrm{vox}}=10^{-6}, N=60$, $D=4,40,400 \times 10^{-9}, D_{1}(x)=D_{2}(x)$ if $x \in \Omega_{S}$ and $D_{1}(x)=0$ otherwise.

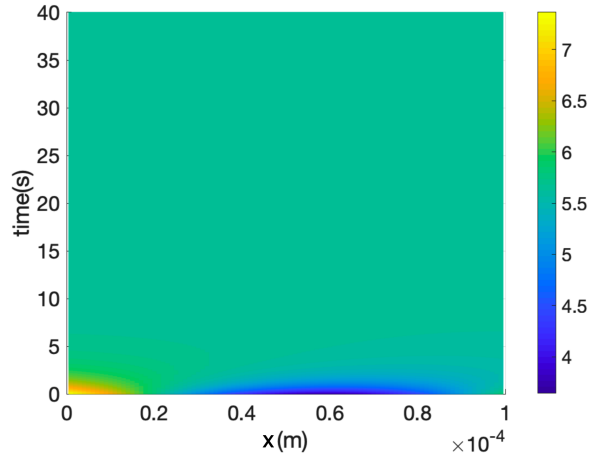

(a) $D_{2}(x)=D /\left(1+10^{4} x\right)$.

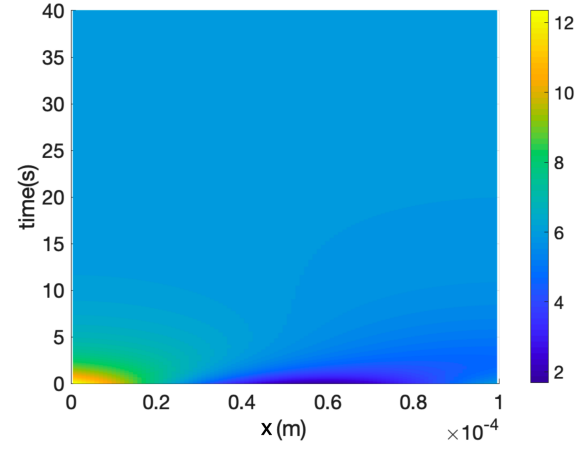

(b) $D_{2}(x)=D\left(1+e^{-\left(\left(10^{4} x-1\right) / 2\right)^{2}}\right)$.

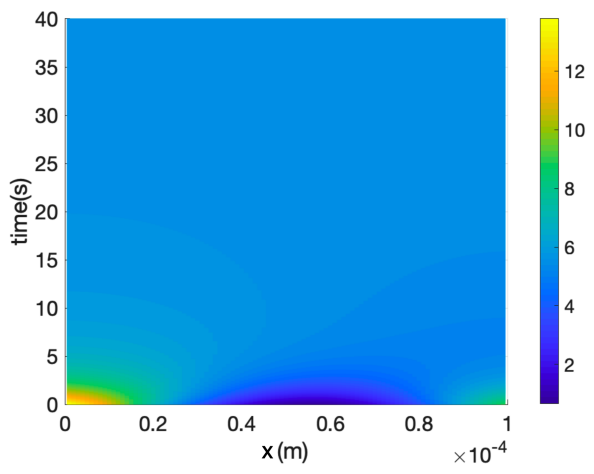

(c) $D_{2}(x)=D+D e^{10^{4}(x)}$.

Fig. 4: Solution of (21) for the concentration of $\mathrm{S}_{2}$ for different $D(x), a^{1}=a^{2}=1 \mathrm{~s}^{-1}, \Delta=600, V_{\mathrm{Rx}}=V_{\mathrm{Tx}}=V_{\text {vox }}=10^{-6}$, $N=100, D=40 \times 10^{-9} \mathrm{~m}^{2} / \mathrm{s}, D_{1}(x)=D_{2}(x)$ if $x \in \Omega_{S}$ and $D_{1}(x)=0$ otherwise.

TABLE II: KS Test for (9) in Assertion 1.

\begin{tabular}{|l|l|l|}
\hline Parameters & Acceptance Probability & $p$-value \\
\hline$\Delta=60, N V_{\text {vox }} / V_{\mathrm{Rx}}=10$ & 0.92 & 0.32 \\
\hline$\Delta=100, N V_{\text {vox }} / V_{\mathrm{Rx}}=10$ & 0.93 & 0.40 \\
\hline$\Delta=600, N V_{\text {vox }} / V_{\mathrm{Rx}}=10$ & 0.94 & 0.46 \\
\hline$\Delta=100, N V_{\text {vox }} / V_{\mathrm{Rx}}=20$ & 0.92 & 0.40 \\
\hline$\Delta=600, N V_{\text {vox }} / V_{\mathrm{Rx}}=20$ & 0.92 & 0.43 \\
\hline$\Delta=600, N V_{\text {vox }} / V_{\mathrm{Rx}}=100$ & 0.91 & 0.43 \\
\hline
\end{tabular}

In our setting, we compare the observations at the receiver with the Gaussian law in Assertion 1 using the KolmogorovSmirnov test for $U=500$. Table II shows the results of the test with a confidence of $\alpha=0.05$. As can be seen in this table, data obtained from the particle-based simulations is in good agreement Assertion 1 with high acceptance rate and $p$ values significantly larger than the confidence level $\alpha=0.05$, which suggests that Assertion 1 cannot be ruled out.

The second part of Assertion 1 corresponding to (10) states that near equilibrium the quantity of $S_{1}$ and the quantity of $\mathrm{S}_{2}$ in the receiver are approximately independent. In order to verify this part of the assertion, we have performed an additional KS test. Table 1 shows the results of the test with confidence level $\alpha=0.05$ for varying reaction rates $a^{1}, a^{2}$. As for Table II, the results show a high acceptance rate and high $p$-values, indicating that (10) cannot be ruled out.
TABLE III: KS Test for (10) in Assertion 1.

\begin{tabular}{|l|l|l|}
\hline Parameters & Acceptance Probability & $p$-value \\
\hline$\Delta=100, N V_{\text {vox }} / V_{\mathrm{Rx}}=10,\left(\frac{a^{1}}{a^{2}}\right)=1$ & 0.89 & 0.3 \\
\hline$\Delta=100, N V_{\text {vox }} / V_{\mathrm{Rx}}=10,\left(\frac{a^{1}}{a^{2}}\right)=2$ & 0.88 & 0.32 \\
\hline$\Delta=600, N V_{\text {vox }} / V_{\mathrm{Rx}}=10,\left(\frac{a^{1}}{a^{2}}\right)=1$ & 0.91 & 0.34 \\
\hline$\Delta=600, N V_{\text {vox }} / V_{\mathrm{Rx}}=20,\left(\frac{a^{1}}{a^{2}}\right)=2$ & 0.90 & 0.34 \\
\hline
\end{tabular}

\section{System Parameter Design}

In this section, we focus on the design of key system parameters including the sampling time, the detection threshold in the low memory scheme from Sec. III-B. We also develop an estimation procedure for the container volume, which is necessary for selecting the decision threshold.

\section{A. Choosing the Sampling Time}

The equilibrium signaling scheme is based on the assumption that sampling is performed when the system is nearly in equilibrium. As such, we now turn to the problem of selecting the sampling time. We base the analysis on the underlying deterministic system in (21), which determines the average behavior of the system governed by the RDME.

As a tractable closed-form solution to (21) is not available, we introduce a heuristic approach which provides a means 
of selecting the sampling time. The analysis is based on a one dimensional model with a spatially homogeneous diffusion coefficient for $S_{2}$. This is in order to obtain a simple heuristic in order to obtain a sampling time. We also assume that only $S_{1}$ is observed by the receiver, although the analysis can be carried out analogously for observations of both $S_{1}$ and $S_{2}$.

Our approach decomposes the kinetics into three phases: a reaction-limited phase in the transmitter; diffusion of $S_{2}$ from the transmitter to the receiver; and a reaction-limited phase in the receiver. Each phase is formalized in the following, where we assume $a^{1}=a^{2}=a$ and $V_{\mathrm{Tx}}=V_{\mathrm{Rx}}$.

a) Phase A: In the first phase, the system is modeled as a single container with reaction $S_{1} \rightarrow S_{2}$ and no diffusion. In particular, the initial concentration of $\mathrm{S}_{l}$ is denoted by $u_{A, l}^{0}$, with $u_{A, 1}^{0}=\Delta$ and $u_{A, 2}^{0}=0$, and the concentrations evolve according the following differential equation

$$
\frac{\mathrm{d} u_{A, 1}}{\mathrm{~d} t}=-a u_{A, 1}(t)
$$

which admits the explicit solution

$$
u_{A, 1}(t)=u_{A, 1}(0) e^{-a t}=\Delta e^{-a t} .
$$

It is clear that $\lim _{t \rightarrow \infty} u_{A, 1}(t)=0$. On the other hand, for sufficiently small $\epsilon$, we can obtain the approximate equilibrium time for the first phase, $t_{A}$, by plugging $u_{A, 1}(t)=\epsilon$ into (25) as

$$
t_{A}=-\frac{1}{a^{1}} \log \left(\frac{\epsilon}{\Delta}\right) .
$$

b) Phase B: In the second phase, a diffusion-limited model is adopted, where chemical reactions are ignored. In this case, the concentration evolves according to

$$
\frac{\partial u_{B, 2}}{\partial t}(x, t)=D \frac{\partial^{2} u_{B, 2}}{\partial x^{2}}(x, t),
$$

where $u_{2, B}(x, 0)=\Delta \delta_{x=0}$, with $\delta_{x=0}$ denoting the Dirac delta function. The solution to this differential equation is given by

$$
u_{B, 2}(x, t)=\frac{\Delta}{\sqrt{4 \pi D t}} \exp \left(-\frac{x^{2}}{4 D t}\right) .
$$

Since at equilibrium, spatial homogeneity is required, the spatial derivative in (28) should be negligible. This implies that $L^{2} \ll 4 D t$ where $L$ is chosen to be the maximum distance between the transmitter and the container. Therefore, the required time for spatial homogeneity, $t_{B}$, can be expressed as

$$
\frac{L^{2}}{4 D} \ll t_{B}
$$

c) Phase C: In the third phase, the system is again modeled as a single container with $\mathrm{S}_{2} \rightarrow \mathrm{S}_{1}$ and no diffusion. As in Phase $\mathrm{A}$, the evolution of the concentrations $u_{C, 2}$ is governed via (24), with $u_{C, 2}$ in place of $u_{A, 1}$ and initial conditions given by $u_{C, 2}^{0}=\Delta V_{\mathrm{Rx}} /\left(N V_{\text {vox }}\right)$ (due to spatial homogeneity in Phase B) and $u_{C, 1}^{0}=0$. Hence,

$$
u_{C, 2}(t)=\frac{\Delta V_{\mathrm{Rx}}}{\left(N V_{\mathrm{vox}}\right)} e^{-a t} .
$$

Note that, as discussed in Section III, the expected number of molecules in the receiver in equilibrium is $\mu_{r, 1}=$ $\frac{\Delta V_{\mathrm{Rx}}}{N V_{\mathrm{vox}}+2 V_{\mathrm{Rx}}}$. The required time $t_{C}$ to decrease from $\Delta V_{\mathrm{Rx}} /\left(N V_{\text {vox }}\right)$ to $\frac{\Delta V_{\mathrm{Rx}}}{N V_{\text {vox }}+2 V_{\mathrm{Rx}}}$ can be obtained by using (30) as

$$
t_{C}=-\frac{1}{a^{2}} \log \left(\frac{N V_{\mathrm{vox}}}{N V_{\mathrm{vox}}+2 V_{\mathrm{Rx}}}\right) .
$$

Let, $t_{r}=t_{A}+t_{B}$ and $t_{d}=t_{C}$ denote the required time to approach equilibrium. A useful heuristic for the required time to approach equilibrium is then given by

$$
t^{*}=t_{r}+t_{d}
$$

which is plotted in Fig. 5 and evaluated for different parameters in Table IV. In the table, $D_{l}(x)=D_{2}(x)=D$, $\epsilon=10^{-3}, r=N V_{\mathrm{vox}} / V_{\mathrm{Rx}}$. The time $t_{e q}$ corresponds to when the solution to (21) is first within $\epsilon$ of the equilibrium concentration. In order to calculate the $t_{e q}$, the system (21) is solved numerically.

Observe that with the realistic parameters in Fig. 5, equilibrium is approximately reached before $50 \mathrm{~s}$. In general, the rate of convergence depends on the diffusion coefficients, reaction rate coefficients, and also the initial quantity of molecules for each species. Therefore, these parameters should be carefully considered in the design of the communication system in order to match the desired symbol period.

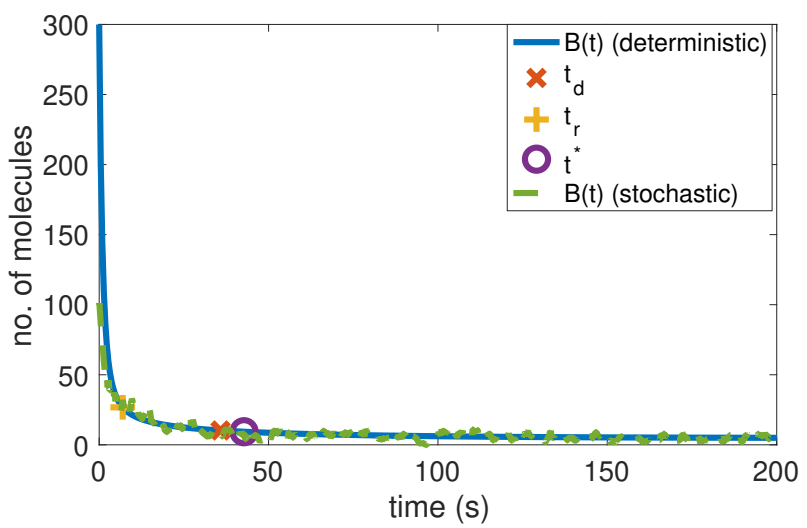

Fig. 5: Value of $t^{*}$ in (32) for $D=80 \times 10^{-11} \mathrm{~m}^{2} / \mathrm{s}, r=60$, $a^{1}=a^{2}=a=1 \mathrm{~s}^{-1}$.

\section{B. Optimizing the Threshold for Low Memory Detection}

A key parameter for implementing the low memory detection scheme in Section III is the decision threshold. This parameter can be obtained by minimizing the probability of error, defined by

$$
\begin{aligned}
P_{e}^{n, m}(\tau)= & \frac{1}{2}\left(\operatorname{Pr}\left(R(n)>\tau \mid s_{n}=0\right)\right. \\
& \left.+\operatorname{Pr}\left(R(n) \leq \tau \mid s_{n}=1\right)\right),
\end{aligned}
$$


TABLE IV: Sampling Time Heuristic $t_{e q}$ for $\epsilon=0.01$.

\begin{tabular}{|l|l|l|l|l|}
\hline Parameters & $t_{B}$ & $t_{A}+t_{C}$ & $t^{*}$ & $t_{e q}$ \\
\hline$D=80 \times 10^{-9} \mathrm{~m}^{2} / \mathrm{s}, r=60, a=0.1 \mathrm{~s}^{-1}$ & $0.3 \mathrm{~s}$ & $74.3 \mathrm{~s}$ & $74.6 \mathrm{~s}$ & $80.1 \mathrm{~s}$ \\
\hline$D=80 \times 10^{-10} \mathrm{~m}^{2} / \mathrm{s}, r=60, a=0.1 \mathrm{~s}^{-1}$ & $3.6 \mathrm{~s}$ & $74.3 \mathrm{~s}$ & $77.9 \mathrm{~s}$ & $82 \mathrm{~s}$ \\
\hline$D=80 \times 10^{-11} \mathrm{~m}^{2} / \mathrm{s}, r=60, a=0.1 \mathrm{~s}^{-1}$ & $36 \mathrm{~s}$ & $74.3 \mathrm{~s}$ & $110.3 \mathrm{~s}$ & $118 \mathrm{~s}$ \\
\hline$D=80 \times 10^{-11} \mathrm{~m}^{2} / \mathrm{s}, r=60, a=1 \mathrm{~s}^{-1}$ & $36 \mathrm{~s}$ & $7.4 \mathrm{~s}$ & $43.4 \mathrm{~s}$ & $50.9 \mathrm{~s}$ \\
\hline$D=80 \times 10^{-11} \mathrm{~m}^{2} / \mathrm{s}, r=100, a=1 \mathrm{~s}^{-1}$ & $99.4 \mathrm{~s}$ & $7.4 \mathrm{~s}$ & $106.8 \mathrm{~s}$ & $114 \mathrm{~s}$ \\
\hline
\end{tabular}

where $n$ is the symbol index and $m$ is the number of previously transmitter symbols corresponding to bit 1 . This probability of error is evaluated in Proposition 1.

Proposition 1. Assume that the previous $n-1$ symbols have been correctly decoded, with $m$ transmissions of bit 1 . Then, under Assertion 1, the low memory detector in (17) has a probability of error for the n-th symbol given by

$$
\begin{aligned}
P_{e}^{n, m}(\tau) & =0.5\left(1-Q\left(\frac{\tau-\mu_{r}}{\sqrt{(m+1) \mu_{r}+m \mu_{r}}}\right)\right) \\
& +0.5 Q\left(\frac{\tau}{\sqrt{2 m \mu_{r}}}\right),
\end{aligned}
$$

where $\mu_{r}=\mu_{r, 1}$ if only $S_{1}$ is observed by the receiver, and $\mu_{r}=\mu_{r, 1}+\mu_{r, 2}$ if both $\mathrm{S}_{1}$ and $\mathrm{S}_{2}$ are observed. We also denote $Q(x)=\int_{x}^{\infty} \frac{1}{\sqrt{2 \pi}} \exp \left(-\frac{z^{2}}{2}\right) \mathrm{d} z, x \in \mathbb{R}$.

Proposition 1 follows immediately from the Gaussian statistics in Assertion 1. Note that it is necessary to index $P_{e}^{n, m}$ by $n$ and $m$ due to the fact that the receiver observation statistics vary as $n$ and $m$ increase. As such, the optimal threshold also depends in general on $n$ and $m$. by

To proceed, we note that the derivative of $P_{e}^{n, m}(\tau)$ is given

$$
\begin{aligned}
\frac{\mathrm{d} P_{e}^{(n, m)}}{\mathrm{d} \tau} & =\frac{1}{\sqrt{2 \pi(2 m+1) \mu_{r}}} \exp \left(-\frac{\left(\tau-\mu_{r}\right)^{2}}{2(2 m+1) \mu_{r}}\right) \\
& -\frac{1}{\sqrt{4 \pi m \mu_{r}}} \exp \left(-\frac{\tau^{2}}{4 m \mu_{r}}\right) .
\end{aligned}
$$

Therefore, for large $m$,

$$
\begin{aligned}
\frac{\mathrm{d} P_{e}^{(n, m)}}{\mathrm{d} \tau} & \approx-\frac{1}{\sqrt{4 \pi m \mu_{r}}} \exp \left(-\frac{\tau^{2}}{4 m \mu_{r}}\right) \\
& +\frac{1}{\sqrt{4 \pi m \mu_{r}}} \exp \left(-\frac{\left(\tau-\mu_{r}\right)^{2}}{4 m \mu_{r}}\right) .
\end{aligned}
$$

The threshold minimizing the probability of error for large $m$ can then be well-approximated by

$$
\tau^{*}=\frac{\mu_{r}}{2} .
$$

We remark for a sufficiently large number of transmissions, even if some symbols have been incorrectly decoded, $\tau^{*}$ in (35) is a good approximation for the optimal threshold for the low memory detector. Then the optimum $P_{e}^{n, m}$ can be obtained as

$$
\begin{aligned}
P_{e}^{n, m}\left(\tau^{*}\right) & =0.5\left(1-Q\left(\frac{-\frac{\mu_{r}}{2}}{\sqrt{(m+1) \mu_{r}+m \mu_{r}}}\right)\right) \\
& +0.5 Q\left(\frac{\frac{\mu_{r}}{2}}{\sqrt{2 m \mu_{r}}}\right)
\end{aligned}
$$

\section{Selection of Receiver Observations}

So far, we have allowed the possibility of the receiver observing only $S_{1}$ or both $S_{1}$ and $S_{2}$. From Assertion 1, it is clear that this decision depends on the reaction rates $a^{1}, a^{2}$. In particular, the probability of error in (36) is a decreasing function of $\mu_{r}$.

It is also possible to consider equilibrium signaling with only a single species and no chemical reactions. This scenario is analogous to standard CSK schemes, where observations are made near equilibrium. In this case, the expected number of molecules in the receiver at the sampling time after a single bit- 1 transmission is given by

$$
\mu_{C S K}=\Delta \frac{V_{\mathrm{Rx}}}{V_{t o t}} .
$$

A comparison of Assertion 1 and (37) reveals that if the reaction rates $a^{1}$ and $a^{2}$ are equal, then $\mu_{C S K}>\mu_{r, 1}$. On the other hand, as $\left(\frac{a^{1}}{a^{2}}\right)$ increases, so does $\mu_{r, 1}$. However, this is not the case for $\mu_{C S K}$. As such, for sufficiently large $\left(\frac{a^{1}}{a^{2}}\right)$, $\mu_{r, 1}>\mu_{C S K}$, yielding a lower probability of error. If both $\mathrm{S}_{1}$ and $\mathrm{S}_{2}$ are observed at the receiver, then if $a^{1}=a^{2}$, $\mu_{r, 1}+\mu_{r, 2}=2 \mu_{C S K}>\mu_{C S K}$. As such, for $a^{1} \approx a^{2}$, it is desirable to observe both $S_{1}$ and $S_{2}$. These observations are illustrated in Fig. 6. In particular, we see the significant advantage of observing both $\mathrm{S}_{1}$ and $\mathrm{S}_{2}$ (corresponding to $\mu_{r, 1}+\mu_{r, 2}$ ) over utilizing a single chemical species in the system (corresponding to $\mu_{C S K}$ ).

\section{Container Volume Estimation}

The two key parameters in equilibrium signaling are the relative volumes of the transmitter and receiver with respect to the total container volume. For applications in vitro, for example, the total volume the container and the relative volumes of the transmitter and receiver may not be known a priori. This is due to the fact that the exact environment of the molecular communication system may be complex or time varying. As such, it is highly desirable to estimate the container volume.

Unlike more detailed features of the container-which are required to optimize decisions in classical CSK-estimating 


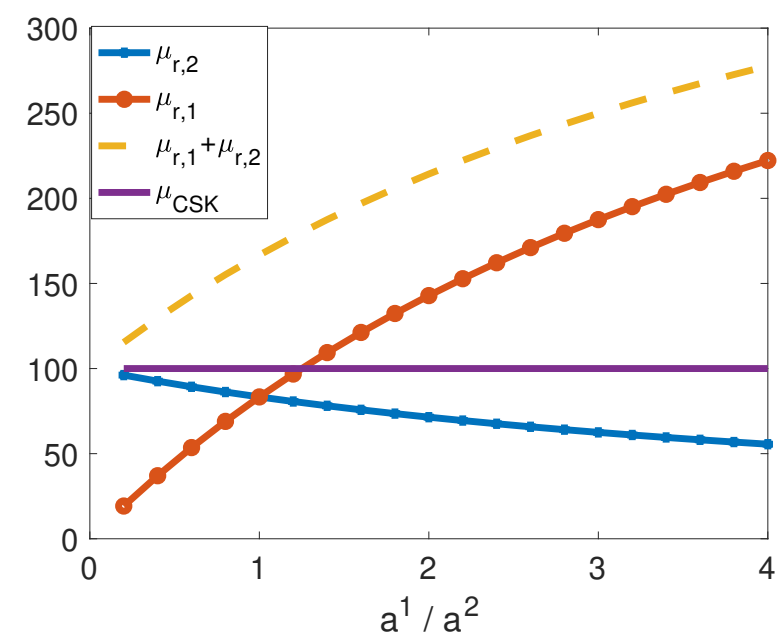

Fig. 6: Impact of $\frac{a^{1}}{a^{2}}$ on $\mu_{r, 1}$ and $\mu_{r, 2}$ with $\Delta=1000, V_{\mathrm{Tx}}=$ $V_{\mathrm{Rx}}=\frac{V_{t o t}}{10}$.

the container volume is straightforward. Suppose that $K$ transmissions of $\Delta$ molecules of $S_{1}$, corresponding to $K$ time slots, are allocated to volume estimation. Under Assertion 1, the observations $x_{1}, \ldots, x_{K}$ of the quantity of $\mathrm{S}_{1}$ near equilibrium are independent with known Gaussian statistics. In particular, the variance of sample $k$ is then given by $k \mu_{r}$, where $\mu_{r}$ is given by (9).

Under Assertion 1, the observations are Gaussian and therefore the maximum likelihood estimator $\hat{\mu}_{r}$ for $\mu_{r}$ is the solution to

$$
\hat{\mu}_{r}=\arg \max _{\mu_{r}} \prod_{k=1}^{K} \frac{1}{\sqrt{2 \pi k \mu_{r}}} \exp \left(-\frac{\left(x_{k}-k \mu_{r}\right)^{2}}{2 k \mu_{r}}\right),
$$

which admits a solution satisfying

$$
\sum_{k=1}^{K} \frac{k}{2}+\sum_{k=1}^{K} \frac{1}{2 \hat{\mu}_{r}}+\frac{1}{2} \sum_{k=1}^{K} \frac{x_{k}^{2}}{2 k \hat{\mu}_{r}^{2}}=0 .
$$

While the objective in (38) is in general non-convex, it is twice differentiable and therefore it is straightforward to verify numerically which of the solutions corresponds to a maximum.

An expression for the relationship between $\mu_{r}$ and the total volume of the container $N V_{\text {vox }}$ is given in (9). Using this relationship, the estimator for the volume of the container $N V_{\text {vox }}$ is given by

$$
N V_{\mathrm{vox}}=\frac{\Delta V_{\mathrm{Rx}}-\hat{\mu}_{r} \frac{a^{1}}{a^{2}}\left(V_{\mathrm{Tx}}+V_{\mathrm{Rx}}\right)}{\hat{\mu}_{r}} .
$$

Fig. 7 shows the impact of increasing the number of samples on the normalized mean-square error (NMSE). Observe that using (39), it is possible to estimate the volume of the container with low NMSE even for small numbers of samples and regardless of the true value of $\mu_{r}$.

\section{NUMERICAL RESULTS}

In this section, we study the performance of the proposed equilibrium signaling scheme via particle-based simulations.

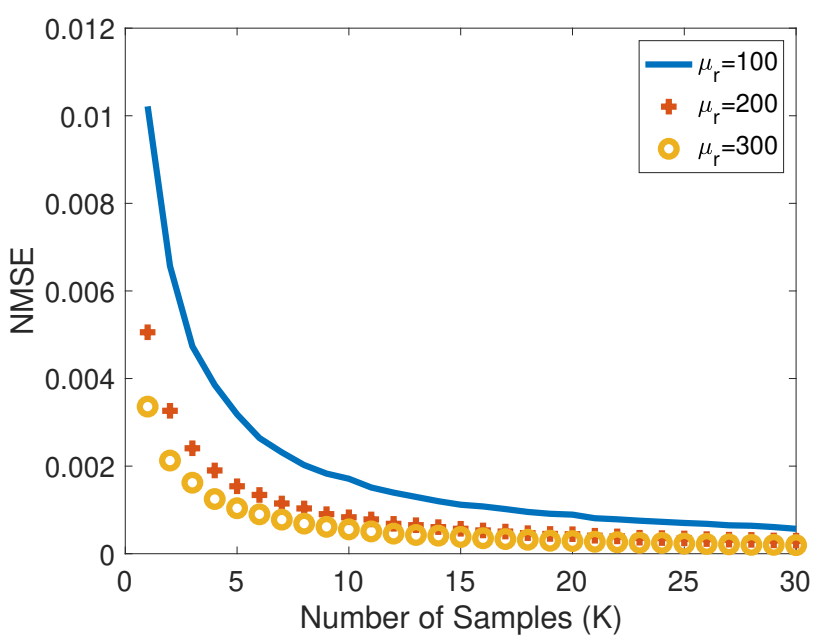

Fig. 7: Plot of NMSE for varying numbers of samples and $\mu_{r}$.

Throughout this section, we assume that the receiver can observe both molecules of $S_{1}$ and $S_{2}$.

Both the near-optimal and low memory schemes are compared, along with a scheme based on CSK. These comparisons are based on transmissions of $n=1000$ bits. Since the channel is non-stationary, the performance is evaluated in terms of the average number of errors in the sequence of $n$ bits. More formally, let $E_{i}$ be the error random variable for bit $i$ in the sequence; that is

$$
E_{i}= \begin{cases}1 & \hat{s}_{i} \neq s_{i} \\ 0 & \hat{s}_{i}=s_{i}\end{cases}
$$

where $\hat{s}_{i}$ is the estimate of the transmitted bit $s_{i}$. Then, the average probability of error is defined as

$$
P_{\text {ave }}=\mathbb{E}\left[\frac{1}{n} \sum_{i=1}^{n} E_{i}\right]
$$

In order to estimate $P_{\text {ave }}, 10000$ iterations of the transmission of $n$ bits are simulated.

The parameters used in the simulations are: $a^{1}=1,2,3 \mathrm{~s}^{-1}$, $a^{2}=1 \mathrm{~s}^{-1} ; D_{1}=D_{2}=80 \times 10^{-11} \mathrm{~m}^{2} / \mathrm{s} ; T_{s}=t^{*}$ given in (32); and $V_{\mathrm{Rx}}=V_{\mathrm{Tx}}$. We remark that very similar results are obtained with different choices of $a^{1}, a^{2}, D_{1}, D_{2}$ as long as the ratio $a^{1} / a^{2}$ remains constant. This is due to the fact that $\mu_{r}=\mu_{r, 1}+\mu_{r, 2}$ only depends on the ratio and not the precise values of $a^{1}$ and $a^{2}$.

In the numerical results, five scenarios are considered by using channel in Fig. 2a:

(i) Near-optimal detection scheme: The average probability of error for Viterbi-based detection scheme developed in Sec. III-A is obtained via particle-based simulations. In particular, the dynamics arising from the RDME model are simulated using the next reaction algorithm [40].

(ii) Low memory detection scheme: The average probability of error for the low memory detection scheme developed in Sec. III-B is obtained via particle-based simulations in the same manner as for the near-optimal scheme. 


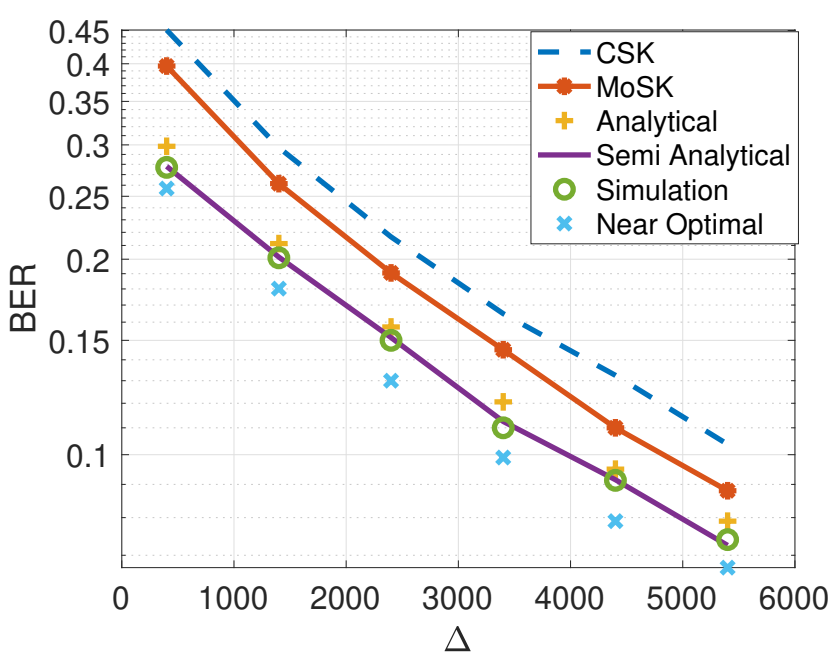

(a) $V_{\mathrm{Rx}} /\left(N V_{\text {vox }}\right)=0.01,\left(\frac{a^{1}}{a^{2}}\right)=1$

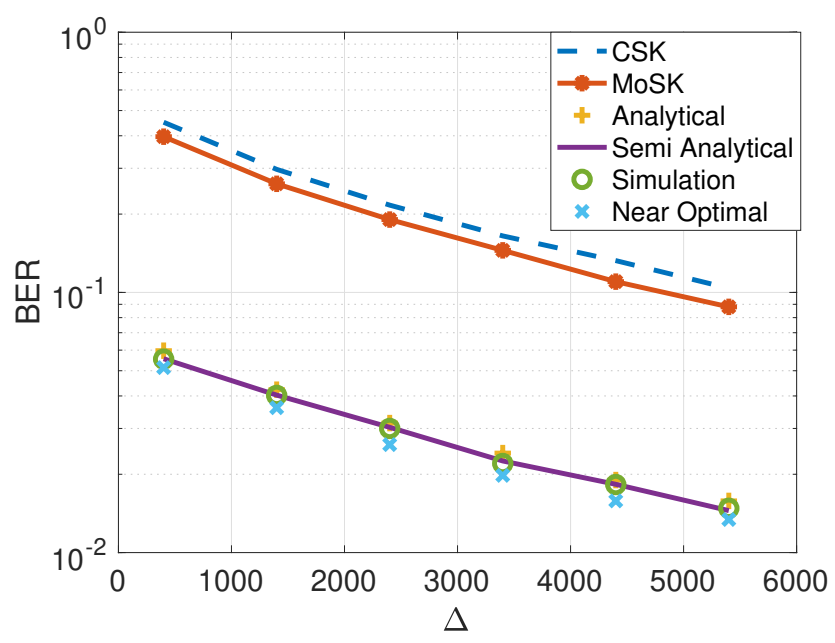

(c) $V_{\mathrm{Rx}} /\left(N V_{\text {vox }}\right)=0.01,\left(\frac{a^{1}}{a^{2}}\right)=2$.

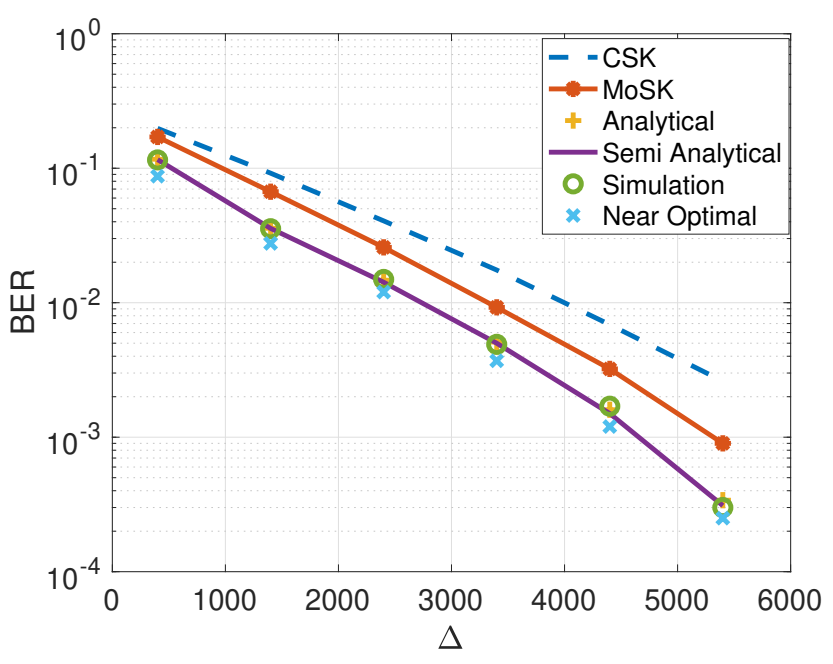

(b) $V_{\mathrm{Rx}} /\left(N V_{\mathrm{vox}}\right)=0.1,\left(\frac{a^{1}}{a^{2}}\right)=1$

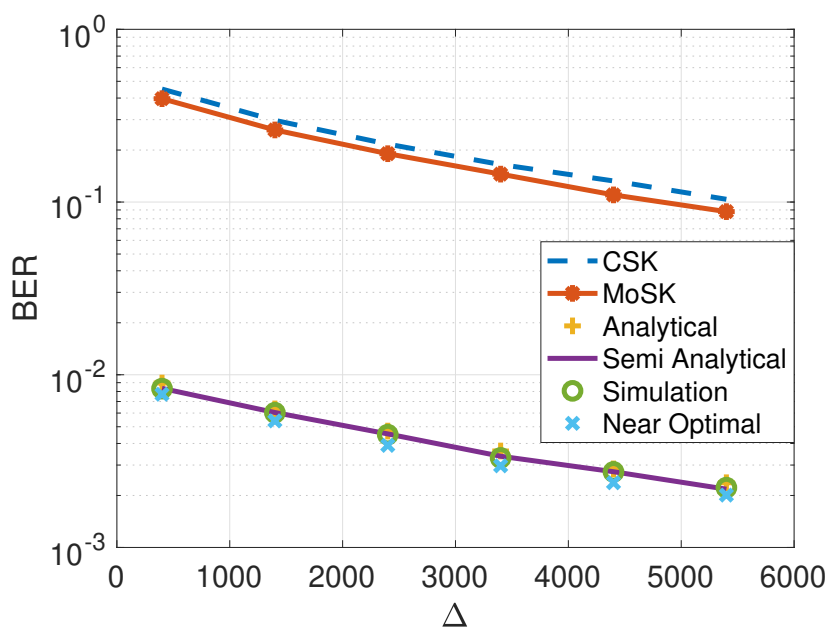

(d) $V_{\mathrm{Rx}} /\left(N V_{\text {vox }}\right)=0.01,\left(\frac{a^{1}}{a^{2}}\right)=3$.

Fig. 8: BER performance for different $V_{\mathrm{Rx}} /\left(N V_{\mathrm{vox}}\right)$ and $\frac{a^{1}}{a^{2}}$.

(iii) Semi-analytical evaluation: For the low memory detection scheme developed in Sec. III-B, the observations in the receiver are simulated based on Assertion 1.

(iv) Analytical evaluation: For the low memory detection scheme developed in Sec. III-B, the probability of error is approximated by the expression in Proposition 1.

(v) Equilibrium CSK: The average probability of error for a scheme where only a single molecule is employed is obtained via particle-based simulations as for the nearoptimal scheme. The sampling time is chosen as $t^{*}$ with observations drawn from $S_{2}$ (the only species in the system), which may be necessary due to limitations of the biological oscillator needed to implement sampling.

Fig. 8 plots the average probability of error for varying quantities of emitted molecules $\Delta$, in each of the five scenarios and with varying receiver and transmitter volumes. As expected, the near-optimal scheme based on the Viterbi algorithm outperforms the low memory scheme. The performance gains depend on the relative volume of the receiver, ranging from approximately 300 molecules in the case where $V_{\mathrm{RX}} /\left(N V_{\mathrm{Vox}}\right)=0.05$. In general, this suggests a tradeoff between the complexity of the receiver and available energy in the transmitter. Fig. 8 also shows that for the low memory scheme, the semi-analytical and analytical models well approximate the results from particle-based simulations. This provides further evidence for the validity of Assertion 1 and also Proposition 1.

Moreover, Fig. 8 shows a significant performance gain using equilibrium signaling over equilibrium CSK under the sampling time constraint. To gain intuition into why this gain arises, observe that near equilibrium the average number of molecules observed in the receiver under CSK (i.e., only $\mathrm{S}_{2}$ is present in the system) will be $\Delta \frac{V_{\mathrm{Rx}}}{N V_{\mathrm{vox}}}$. On the other hand, the proposed equilibrium signaling scheme yields on average a quantity $\mu_{r}=\mu_{r, 1}+\mu_{r, 2}$ given in (9). In any scenario where the number of voxels comprising the channel is greater than zero, $\mu_{r}>\Delta \frac{V_{\mathrm{Rx}}}{N V_{\text {vox }}}$, which is the reason for the performance gains. 
We remark that the gain of equilibrium signaling over CSK may not be present if the sampling time is optimized as in classical CSK. However, realistic biological circuits may place strong constraints on sampling [22] and therefore such a constraint on the sampling time may be unavoidable.

\section{CONCLUSION}

A key challenge for molecular communications, particularly in biological environments, is uncertainty in the geometry of the environment. This uncertainty may take the form of the shape of the environment, or the distance between the transmitter and the receiver. As it is not necessarily straightforward to estimate parameters of the environment and schemes such as classical CSK are not robust to changes in the geometry, it is highly desirable to develop schemes that are in fact robust.

In this paper, we proposed equilibrium signaling, which only requires knowledge of the container volume in order to develop near-optimal detection schemes. This robustness comes at the cost of large sampling times; however, it is necessary in some applications to exploit biological oscillators in order to obtain samples. As such, the requirement of long sampling periods may in fact be a realistic system constraint.

In order to precisely specify conditions under which equilibrium signaling can be applied, we focused on systems satisfying the assumption in Sec. II. Nevertheless, there is strong evidence that some of these assumptions can be relaxed. In the case that no chemical reactions are present (even in the transmitter and receiver), diffusion can be modeled via the Smoluchowski equation, which allows external forces to be accounted for. An initial investigation applying equilibrium signaling in this model has been carried out in [42].

It should be possible to allow additional chemical reactions, as long as an equilibrium state can be guaranteed. For example, with weakly reversible first order reactions or high order reactions satisfying complex balance as well as appropriate choices of the diffusion jump rates [43]. We remark, however, that in the case of high order reactions, nonlinearities in the Fokker-Planck equation make a rigorous characterization of the equilibrium state more challenging.

We also expect that slow degradation of molecules can be accounted for within the equilibrium signaling framework. We expect that analysis of this situation can be carried out within the quasi-equilibrium framework commonly used to investigate enzyme-based reactions (see, e.g., [44]). A related issue is the sensitivity of the receiver in the presence of very large quantities of information-carrying molecules. This may be accounted for by limiting the number of consecutive transmissions and incorporating a cleaning process to remove information-carrying molecules from the system.

Finally, we have focused on utilizing only a single chemical species within the channel. Nevertheless, an interesting question is whether equilibrium signaling exploiting multiple molecules in the channel (such as in MoSK [19], MCSK [2], D-MoSK [20]) brings significant performance improvements.

\section{REFERENCES}

[1] W. Coffey, Y. Kalmykov, and J. Waldron, The Langevin Equation. World Scientific, 2004.
[2] H. Arjmandi, A. Gohari, M. Kenari, and F. Bateni, "Diffusion-based nanonetworking: a new modulation technique and performance analysis," IEEE Communications Letters, vol. 17, no. 4, pp. 645-648, 2013.

[3] B. Atakan and O. Akan, "On channel capacity and error compensation in molecular communication," Transactions on Computational Systems Biology X, vol. 10, pp. 59-80, 2008.

[4] — "Deterministic capacity of information flow in molecular nanonetworks," Nano Communication Networks, vol. 1, no. 1, pp. 31-42, 2010.

[5] M. Mahfuz, D. Makrakis, and H. Mouftah, "Spatiotemporal distribution and modulation schemes for concentration-encoded medium-to-long range molecular communications," in 25th Biennial Symp. Commun., 2010.

[6] I. Llatser, A. Cabellos-Aparicio, M. Pierobon, and E. Alarcon, "Detection techniques for diffusion-based molecular communnication," IEEE Journal on Selected Areas in Communications, vol. 31, no. 12, pp. 726734, 2013.

[7] N.-R. Kim and C.-B. Chae, "Novel modulation techniques using isomers as messenger molecules for nano communication networks via diffusion," IEEE Journal of Selected Areas in Communications, vol. 31, no. 12 , pp. 847-856, 2013.

[8] M. Mahfuz, D. Makrakis, and H. Mouftah, "A comprehensive study of sampling-based optimum signal detection in concentration-encoded molecular communication," IEEE Transactions on NanoBioscience, vol. 13 , no. 3, pp. 208-222, 2014.

[9] — - "Concentration-encoded subdiffusive molecular communication: theory, channel characteristics, and optimum signal detection," IEEE Transactions on NanoBioscience, vol. 15, no. 6, pp. 533-548, 2016.

[10] M. Kuran, H. Yilmaz, T. Tugcu, and I. Akyildiz, "Modulation techniques for communication via diffusion in nanonetworks," in IEEE International Conference on Communications (ICC), 2011.

[11] _ - "Interference effects on modulation techniques in diffusion based nanonetworks," Nano Communication Networks, vol. 3, no. 1, pp. 6573, 2012.

[12] H. B. Yilmaz, A. C. Heren, T. Tugcu, and C.-B. Chae, "Threedimensional channel characteristics for molecular communications with an absorbing receiver," IEEE Communications Letters, vol. 18, no. 6, pp. 929-932, 2014.

[13] Y. Deng, A. Noel, M. Elkashlan, A. Nallanathan, and K. C. Cheung, "Modeling and simulation of molecular communication systems with a reversible adsorption receiver," IEEE Transactions on Molecular, Biological and Multi-Scale Communications, vol. 1, no. 4, pp. 347-362, 2015.

[14] Y. Deng, A. Noel, W. Guo, A. Nallanathan, and M. Elkashlan, "Analyzing large-scale multiuser molecular communication via 3-d stochastic geometry," IEEE Transactions on Molecular, Biological and Multi-Scale Communications, vol. 3, no. 2, pp. 118-133, 2017.

[15] X. Wang, M. Higgins, and M. Leeson, "Distance estimation schemes for diffusion based molecular communication systems," IEEE Cотmunications Letters, vol. 19, no. 3, pp. 399-402, 2015.

[16] V. Jamali et al., "Channel modeling for diffusive molecular communication-a tutorial review," Proceedings of the IEEE, vol. 107, no. 7, pp. 1256-1301, 2019.

[17] P. Maini, D. Benson, and J. Sherrat, "Pattern formation in reactiondiffusion models with spatially inhomogeneous diffusion coefficients," IMA Journal of Mathematics Applied in Medicine and Biology, vol. 9, pp. 197-213, 1992.

[18] H. ShahMohammadian, G. Messier, and S. Magierowski, "Optimum receiver for molecule shift keying modulation in diffusion-based molecular communication channels," Nano Communication Networks, vol. 3, no. 3, pp. 183-195, 2012.

[19] M. S. Kuran, H. B. Yilmaz, T. Tugcu, and I. F. Akyildiz, "Modulation techniques for communication via diffusion in nanonetworks," in 2011 IEEE international conference on communications (ICC). IEEE, 2011, pp. $1-5$.

[20] M. H. Kabir, S. R. Islam, and K. S. Kwak, "D-mosk modulation in molecular communications," IEEE transactions on nanobioscience, vol. 14 , no. 6, pp. 680-683, 2015.

[21] A. Hamdan and C. Chou, "Generalized solution for the demodulation of reaction shift keying signals in molecular communication networks," IEEE Transactions on Communications, vol. 65, no. 2, pp. 715-727, 2016.

[22] E. Shitiri, A. Vasilakos, and H. Cho, "Biological oscillators in nanonetworks-opportunities and challenges," Sensors, vol. 18, no. 5, 2018.

[23] V. Jamali, N. Farsad, R. Schober, and A. Goldsmith, "Diffusive molecular communications with reactive molecules: channel modeling and 
signal design," IEEE Transactions on Molecular, Biological and MultiScale Communications, vol. 4, no. 3, pp. 171-188, 2018.

[24] A. Noel, K. Cheung, and R. Schober, "Improving receiver performance of diffusive molecular communication with enzymes," IEEE Transactions on NanoBioscience, vol. 13, no. 1, pp. 31-43, 2014.

[25] N. van Kampen, Stochastic Processes in Physics and Chemistry. Elsevier Science \& Technology Books, 2007.

[26] L. Zeman and A. Zydney, Microfiltration and Ultrafiltration: Principles and Applications. CRC Press, 2017.

[27] Y. Chahibi, M. Pierobob, S. Song, and I. Akyildiz, "A molecular communication system model for particulate drug delivery systems," IEEE Transactions on Biomedical Engineering, vol. 60, no. 12, pp. 3468-3483, 2013

[28] N.-R. Kim, A. Eckford, and C.-B. Chae, "Symbol interval optimization for molecular communication with drift," IEEE Transactions on NanoBioscience, vol. 13, no. 3, pp. 223-229, 2014

[29] Y. Fang, A. Noel, A. Eckford, and R. Kennedy, "Symbol-by-symbol maximum likelihood detection for cooperative molecular communication," IEEE Transactions on Communications, vol. 67, no. 7, pp. 48854899, 2019.

[30] A. Singhal, R. Mallik, and B. Lall, "Performance analysis of amplitude modulation schemes for diffusion-based molecular communication," IEEE Transactions on Wireless Communications, vol. 14, no. 10, pp. 5681-5691, 2015.

[31] A. Gyorgy and D. Del Vecchi, "Limitations and trade-offs in gene expression due to competition for shared cellular resources," in IEEE Conference on Decision and Control, 2014.

[32] S. Isaacson, "The reaction-diffusion master equation as an asymptotic approximation of diffusion to a small target," SIAM Journal on Applied Mathematics, vol. 70, no. 1, pp. 77-111, 2009.

[33] C. Chou, "Extended master equation models for molecular communication networks," IEEE Transactions on NanoBioscience, vol. 12, no. 2, pp. 79-92, 2013.

[34] S. Ethier and T. Kurtz, Markov Processes: Characterization and Convergence. John Wiley \% Sons, 2009.

[35] T. Kurtz, "The relationship between stochastic and deterministic models for chemical reactions," The Journal of Chemical Physics, vol. 57, no. 7, pp. 2976-2978, 1972.

[36] D. Gillespie, "The chemical Langevin equation," The Journal of Chemical Physics, vol. 113, no. 1, pp. 297-306, 2000.

[37] L. Cardelli, M. Kwiatkowska, and L. Laurenti, "Stochastic analysis of chemical reaction networks using linear noise approximation," Biosystems, vol. 149, pp. 26-33, 2016.

[38] L. Arnold and M. Theodosoplu, "Deterministic limit of the stochastic model of chemical reactions with diffusion," Advances in Applied Probability, vol. 12, no. 2, pp. 367-379, 1980

[39] M. Egan, B. Q. Tang, and B. C. Akdeniz, "On the input-output relationship for molecular communications in general first-order chemical reaction-diffusion systems," in Proceedings of the Sixth Annual ACM International Conference on Nanoscale Computing and Communication. ACM, 2019, p. 21.

[40] J. Elf, A. Doncic, and M. Ehrenberg, "Mesoscopic reaction-diffusion in intracellular signaling," in Fluctuations and noise in biological, biophysical, and biomedical systems, vol. 5110. International Society for Optics and Photonics, 2003, pp. 114-125.

[41] F. Massey, "The Kolmogorov-Smirnov test for goodness of fit," Journal of the American statistical Association, vol. 46, no. 253, pp. 68-78, 1951.

[42] M. Egan, B. Akdeniz, and B. Tang, "Equilibrium signaling in spatially inhomogeneous diffusion and external forces," 2020.

[43] K. Fellner, W. Prager, and B. Tang, "The entropy method for reactiondiffusion systems without detailed balance: first order chemical reaction networks," Kinetic and Related Models, vol. 10, no. 4, pp. 1055-1087, 2017.

[44] M. Frank, C. Lax, S. Walcher, and O. Wittich, "Quasi-steady state reduction for the Michaelis-Menten reaction-diffusion system," Journal of Mathematical Chemistry, vol. 56, pp. 1759-1781, 2018.

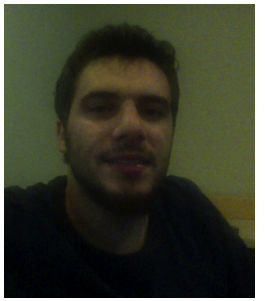

Bayram Cevdet Akdeniz received his B.S. degree in Electronics and Communication Engineering in Yildz Technical University, and the M.Sc. and Ph.D degrees in Electrical-Electronics Engineering from Bogazici University, Istanbul, Turkey, in 2010, 2013 and 2018 respectively. He worked as a post-doctoral researcher in Inria hosted by CITI, a joint laboratory between Inria, INSA Lyon and Université de Lyon, France, between 2018 and 2020. He is currently a post-doctoral researcher at Centre for Bioinformatics at University of Oslo. His research interests are Molecular Communications, Nanonetworks, Communication theory, Bioinformatics and Machine Learning.

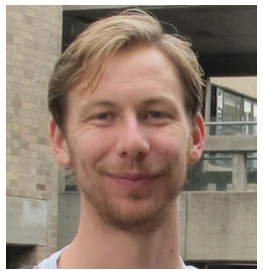

Malcolm Egan (S'10-M'14) received the Ph.D. in Electrical Engineering in 2014 from the University of Sydney, Australia. He is currently a Chargé de Recherche (Permanent Research Staff) in Inria hosted by CITI, a joint laboratory between Inria, INSA Lyon and Université de Lyon, France. Previously he was a Assistant Professor in INSA Lyon, and a Postdoctoral Researcher in the Laboratoire de Mathématiques, Université Blaise Pascal, France and the Department of Computer Science, Czech Technical University in Prague, Czech Republic. $\mathrm{He}$ is currently an Associate Editor for IEEE Communications Letters and previously a guest editor for IEEE Access. His research interests are in the areas of information theory and statistical signal processing with applications in wireless and molecular communications.

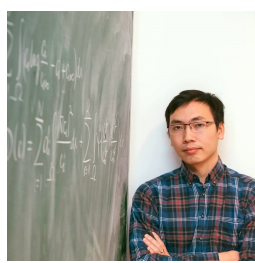

Bao Quoc Tang received his Ph.D. in Mathematics in 2015 from the University of Graz, Austria. Currently, he is a University Assistant (on par with nontenure track Assitant Professor) at the Institute for Mathematics and Scientific Computing, University of Graz. His research includes reaction-diffusion systems arising from chemistry, biology, or physics, and their applications to molecular communications as well as to game theory. 